\title{
Traceable Radiometry Underpinning Terrestrial- and Helio-Studies (TRUTHS): An Element of a Space-Based Climate and Calibration Observatory
}

\author{
Nigel Fox * and Paul Green \\ Earth Observation, Climate and Optical Group, National Physical Laboratory, Hampton Rd, Teddington, \\ Middx TW11 0LW, UK; Paul.green@npl.co.uk \\ * Correspondence: nigel.fox@npl.co.uk
}

Received: 6 July 2020; Accepted: 20 July 2020; Published: 27 July 2020

\begin{abstract}
The Earth's climate is undoubtedly changing; however, the time scale, consequences, and causal attribution remain the subject of significant debate and uncertainty. Detection of subtle indicators from a background of natural variability requires measurements over a time-base of decades. This places severe demands on the instrumentation used, requiring measurements of sufficient accuracy and sensitivity that can allow reliable judgements to be made decades apart. The International System of Units (SI) was developed to address such requirements, providing a reference framework tied to invariant constants of nature. However, ensuring and maintaining SI traceability of sufficient accuracy in instruments orbiting the Earth presents a significant new challenge to the Earth Observation and metrology communities. This paper describes a new satellite mission, called Traceable Radiometry Underpinning Terrestrial- and Helio- Studies (TRUTHS), which enables, for the first time, high-accuracy SI traceability to be established in orbit. The direct use of a 'primary standard' and replication of the terrestrial traceability chain extends the SI into space, in effect realizing a 'metrology laboratory in space' providing and enabling SI-traceable measurements of unequivocal accuracy in the solar reflective domain - an enabling element of an international space-based climate observing system. TRUTHS will not only provide a benchmark of the radiation state of the planet (incoming and outgoing) from which to compare change in the shortest time possible, but also facilitate an upgrade in performance of the Earth Observing system as a whole, through 'in-orbit' reference calibration.
\end{abstract}

Keywords: climate; calibration; SI-traceability; radiometry; solar irradiance; satellite; hyperspectral; radiance

\section{Introduction}

The 21st Conference of the Parties (COP 21) exemplified the intense political, scientific, and public debate about climate change. Observing the changing Earth is at the heart of the world's space agencies agenda, none less so than that of UK space agency (UKSA) and that of its primary partner, European Space Agency, (ESA). Measurements made in support of this observation programme must be universally accepted, robust, and of sufficient accuracy to ensure stability and trust of long-term records. Unfortunately, this must be achieved with an observation system built with technologies known to suffer unpredictable degradation, due to both launch and the harsh environment of space itself. This paper describes a new satellite mission, recently selected to start formal phase A/B1 development in the ESA Earth Watch program, called TRUTHS (Traceable Radiometry Underpinning Terrestrial- and Helio- Studies), which is expressly designed to provide this missing assurance by directly anchoring the space-based Earth observing (EO) systems to the internationally accepted physical standards of the SI measurement system. In this way, guaranteeing the temporal consistency 
of data, even beyond the lifetime of individual systems, whilst simultaneously providing data of unprecedented quality and accuracy to address key challenges across the Earth Science disciplines. This will enhance performance and ensure consistency and coherence of data from the global EO system, including European infrastructure, such as the instruments on-board the Copernicus Sentinels of the European Union, to create a foundation for a unified "operational climate observing system".

The main observational instrument in TRUTHS is a hyperspectral imaging spectrometer (HIS), with continuous spectral extent across the UV-visible-near IR (320 nm to $2400 \mathrm{~nm}$ ) and around $50 \mathrm{~m}$ ground instantaneous field of view (GIFOV), that observes climate-relevant processes related to the atmosphere, oceans, land, and cryosphere and provides new insight for societal understanding of the grand challenges: Food, water, natural resources, health, and disaster management. The relatively high spatial resolution, whilst not essential for many long-term climate observations (larger spatial scales reduce effects of localized anomalies), provides the means to support attribution and mitigation studies and also facilitates robust cross-calibration with a wide range of other optical sensors. The inherent high accuracy and SI-traceability of TRUTHS can then be transferred to the other EO missions leading to an upgrade in performance. Many of the science challenges facing the Earth Observation community lie in the interfaces between disciplines and are driven by uncertainty in our understanding of the Earth system. TRUTHS provides the data to test and constrain climate models and retrieval algorithms upgrading the capability of not only satellite systems, but also surface-based observing systems. In this way, it builds on and enhances the current research programme through injecting new and improved data into proven methods.

The Intergovernmental Panel on Climate Change (IPCC) concludes that the mix of natural variability and anthropogenic effects on decadal timescales is far from fully understood or measured, requiring significant improvements in accuracy [1]. Unequivocal attribution and quantification of subtle fingerprint indicators from this noisy background are fundamental to our ability to predict future climate reliably and to adopt appropriate mitigation/adaptation strategies. The uncertainty in climate prediction (see shaded areas in Figure 1) lies in the complexity of the models; our inadequate understanding of the Earth system; its feedback mechanisms; and the relatively poor quality of available data against which to test predictions on the necessary decadal timescales.

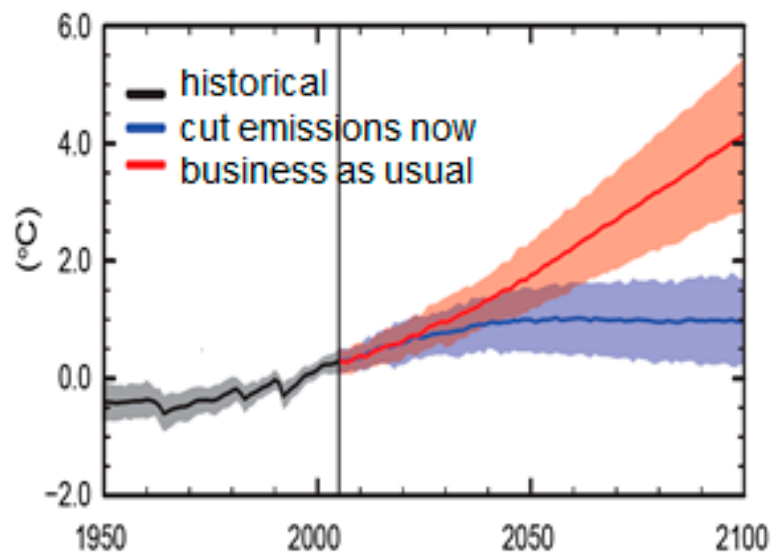

Figure 1. Taken from [1], a plot highlighting variance/uncertainty in the forecasts of temperature rise (shaded) as a result of a specific continued business as usual emission scenario (red) and a net-zero emission from now.

It is in explicitly addressing the concept of uncertainty and seeking climate quality data that differentiates TRUTHS from other complementary hyperspectral imaging spectrometer missions. The TRUTHS payload includes an on-board calibration system, that mimics the Earth-based SI traceability provided at National Measurement Institutes (NMIs). TRUTHS provides on-board radiometric accuracies at 10x that available in current imager sensors. However, importantly, it allows 
continuous re-calibration of the hyperspectral imaging spectrometer (HIS) on-orbit, tied directly to a primary standard, fundamentally maintaining SI traceability for the entire duration of the mission.

The ESA Earth Watch programme facilitates the delivery of Earth observation data for use in operational services. TRUTHS not only delivers an operational global hyperspectral radiance product, but upgrades to 'climate quality' a broad range of operational sensors delivered from across the international space agencies and facilitates the prospect of science quality data from 'newspace' constellations of microsats.

TRUTHS' intrinsic space climate observatory dataset is the science objective that drives the payload performance requirements, but it is in the multi-domain application of the Level 1 (L1) and Level 2 and higher (L2+) datasets of collaborative sensors, fully integrated into the ESA family that cements its true operational credentials.

\section{Mission Concept}

\subsection{Mission Overview}

The concept for space-based SI-traceable reference measurements was developed within the CEOS Working Group on Calibration and Validation (WGCV) and Global Space Intercalibration System (GSICS) of the operational agencies. Multiple space agencies see the need for reliable reference measurements at all the frequencies that are used by instruments to sense the Earth, including visible/near infrared (Vis/NIR), Infrared, and microwave. The principle application area for these measurements, at the highest accuracy levels, is climate; from both the direct sensor measurements and for the calibration of other space-based instruments. However, any application requiring data interoperability, combination of sensors, and other relative information from single scenes, inherently needs to address and understand differences and changes in radiometric accuracy and biases.

To-date two spacecraft mission concepts have emerged to address these top-level goals, TRUTHS, for the solar reflective spectral range ( $320 \mathrm{~nm}-2400 \mathrm{~nm}$ ), led by the UK, and Climate Absolute Radiance and Refractivity Observatory (CLARREO), for the full spectral range, i.e., as TRUTHS but with the addition of thermal IR and no solar measurements, led by NASA, USA. An initial demonstrator mission of CLARREO (Pathfinder) on the International Space Station (ISS) addresses Solar reflective domain down to $\sim 350 \mathrm{~nm}$. So far, no mission has been proposed for the microwave part of the spectrum. More recently China has also announced plans to build a mission to fulfil similar goals. The increased number of such SI-Traceable mission concepts has led to the proposed generic name of SITSATS (SI Traceable Satellites).

SITSATS like TRUTHS and CLARREO are intended to provide highly accurate and trusted climate records and to help constrain the uncertainties in predictions of climate forecast models. The missions are highly complementary and whilst the solar reflective aspect of the two missions seek to fulfil similar objectives, they differ significantly in how the on-board SI-traceability is achieved and also in their native GIFOV, with TRUTHS additionally targeting higher spatial resolution sensors. TRUTHS additionally makes direct measurements of Earth incident solar irradiance, total and spectrally resolved, whereas CLARREO uses the Sun as part of its on-board calibration system. Simultaneous or overlapping flights of the two missions and/or indeed the new proposed Chinese mission, not only provide greater temporal coverage and opportunity to calibrate other EO sensors, but also the means to perform inter-comparisons between themselves to fully validate the uncertainties of the missions and the robustness of the SI scale realized in space. In this way, adopting the same strategy employed by NMIs on the ground to evidence and ensure integrity of the SI system and independent realization of the units.

The rest of this paper will focus solely on TRUTHS—its implementation concept and the application requirements that drive it. It should of course be recognized that many of the user requirements are common to all SITSATS operating in the solar reflective domain and have been derived by extensive 
user consultation and stem from both global climate observing system (GCOS), IPCC, and studies such as the National Research Council (NRC) decadal survey [2].

International government policy for the adaptation to, and mitigation of, climate change, is informed by forecasts of future climate, provided by climate models. The IPCC reporting structure is largely centred on the output of models, indicating the rate of change, effect of competing processes, and the timescales at which various thresholds will be met, under different (emission) scenarios.

All models are approximations of reality, parameterised to allow for limitations in various forms, be that; computational power, understanding of the underlying physics, interactions, and feedbacks; or the extent and availability of observational input data. Even in a scenario with perfect knowledge of all the above, the very stochastic nature of climate means there will be variance in model outputs.

The very nature of the problem means there will be a spread of predictions from an individual model and a spread of predictions between models, due to differences in how the models implement the parameterisations. This variance in model output is unavoidable and is captured as an uncertainty in the forecasts. This uncertainty is costly, and policymakers need to reconcile the economic and political cost of the degree and timeframe of action against immediate political priorities and drivers. In some quarters, this uncertainty in the forecasts is used as evidence to take a limited course of action, or not to act at all.

The uncertainty in, and discrepancies between, models therefore needs to be minimised. At the very heart of the scientific principle, is that models should be tested with quantifiable observations. These observations need as a minimum to enable the principle sources of uncertainties in these models to be tested and in practice these relate to how changes in radiative forcings, feedback to the radiation balance, most notably cloud and albedo. It is of course also critical that the observations can build-up sufficient magnitude above background noise to allow discrimination of any model dependency in as short a time a possible, noting that typical trends are very small and require decades to reach this level and thus in general require multiple time-series of data from different sensors. Robust unequivocal trust in these data sets and their linkage to form harmonised Fundamental Climate Data Records (FCDRs) lies at the heart of the metrology challenge.

\subsection{Mission Objectives}

TRUTHS has two primary objectives. To provide:

1. datasets of sufficient accuracy needed to observationally detect the signals of climate change (in the solar reflective domain) in the shortest possible time. Needed to critically test climate models and attribute the effects of climate change to geophysical processes by providing Top of the Atmosphere (ToA) measurements of incoming and reflected total and spectrally resolved solar radiation appropriately sampled and with uncertainties optimised to minimise time to detect a trend and consistent with goals of GCOS specified requirements.

2. the means to upgrade the performance of the EO system through delivery of an operational high accuracy L1 nadir-looking hyperspectral Earth-reflected radiance product with sufficient spatial $(\sim 50 \mathrm{~m}(\mathrm{Goal}))$, spectral (<8 nm), accuracy (Goal 0.3\%, $\mathrm{k}=2)$, and agility that allows it to match (or address) the observational radiometric characteristics of a wide range of Earth viewing sensors so that it can validate and/or upgrade their calibration by 'reference calibration' (Cal) from space, primarily to address climate measurement needs.

Choice of a 90 degree' precessing orbit provides the opportunity for multiple near simultaneous nadir observations (SNO) and an agile platform compliments this further allowing angle matching so as to remove normal sources of error resulting from view/illumination angles and atmospheric variations. TRUTHS additionally assign's calibration coefficients to existing CEOS/GSICS calibration methods and stable targets such as deserts and the moon enabling not only in-flight sensors to be calibrated, but also back correction of heritage sensors that viewed these targets. Through this reference calibration, SI traceability of the global earth observing system (solar reflective domain) is established; 
robust sensor to sensor interoperability and bias removal achieved (essential for future constellations such as for greenhouse gas (GHG) monitoring, and for many, uncertainty improved, to enable more robust climate quality observations and resilience to potential data gaps.

As a secondary objective, TRUTHS' dataset can be used to provide a source of global hyperspectral data to address a range of applications and, constrain and improve retrieval algorithms.

TRUTHS accuracy has been optimised to be within $20 \%$ of the perfect observing system; i.e., the ratio of the ability to detect a trend from a real observing system with that of the perfect observing system, limited only by natural variability, facilitating the detection of trends in key climate parameters, in as short a time as possible, i.e., to meet objective 1. For example, it will detect a trend in climate change driven cloud radiative forcing (CRF), the dominant and most uncertain climate feedback, in half the time of other sensors with current levels of achievable radiometric accuracy [3].

\subsection{Mission Products and Requirements: Summary}

\subsubsection{Climate 'Top-Level' Requirements}

In the context of the primary objectives, climate, we take from GCOS [4], the specific requirements for radiation balance, in the 'short-wave' domain as these are the most demanding and arguably most urgent. They are presented in Table 1:

Table 1. Global climate observing system (GCOS) requirements driving the radiometric performance drivers of TRUTHS.

\begin{tabular}{ccc}
\hline Parameter & Accuracy & Stability (Decade) \\
\hline Total Solar Irradiance (TSI) & $0.04 \%$ & $0.01 \%$ \\
\hline Solar Spectral Irradiance (SSI) & $0.3 \%(200-2400 \mathrm{~nm})$ & $1 \%$ \\
\hline Reflected solar radiance (integrated) & $1 \mathrm{~W} / \mathrm{m}^{2}=\sim 1 \%$ & $0.3 \mathrm{~W} / \mathrm{m}^{2}=\sim 0.3 \%$ \\
\hline
\end{tabular}

Note: $1 /$ Instantaneous accuracy although demanding, is in the case of TSI $<$ than the requirement for long-term stability. In practice, to have confidence and remove dependencies on a continuous 'overlapping' time-series it is preferable to target the stability requirement; $2 /$ In the context of reflected solar radiance, to aid understanding of attribution, mitigation, and to constrain and test climate forecast models, the reflected solar radiance needs to be spectrally resolved. In particular, for the key radiation feedbacks which drive uncertainty and variance in the models; cloud and albedo and natural carbon sinks such as the oceans and land vegetation.

\subsubsection{Mission Data Products and Performance Requirements}

The mission data products and associated performance requirements derived to achieve the mission objectives are listed in Table 2 and the driving requirements are shown in red.

The most demanding of the driving mission requirements is uncertainty and this stems from TRUTHS' intrinsic space climate observatory dataset applications. For more conventional, instantaneous applications e.g., imagery and to some extent cross-calibration, spectral bandwidth, GIFOV, and SNR (all interdependent) become more critical whilst overall uncertainty can in general be more relaxed.

The evidence for these requirements, derived from user need and the propagation of required uncertainties to the operational geophysical product, is given in the remainder of this section, approached from an operational science area basis. Not all operational science areas are covered, with only key examples, sampled across the disciplines and where the observational characteristics and particularly the accuracy of observations are key drivers for the mission's technical requirements. Further work to explicitly link the full range of user drivers needs to be undertaken, but these are illustrative of those and are driven by those indicated in Table 1, which stem from the GCOS requirements for the corresponding essential climate variables (ECVs).

It should be noted that signal to noise ratio (SNR) values in Table 2 are largely defined to be 'commensurate with the overall uncertainty required by the application and its observational characteristics' and are of course highly dependent on the choice of GIFOV and spectral bandwidth. 
For Earth viewing, they are instantaneous with values given relative to a nominal average Earth albedo of 0.3 and for the corresponding GIFOV specified, for the Sun/Moon it is with respect to several seconds of integration time. Simplistically, the higher the SNR, the smaller the bandwidth and the highest spatial resolution the better but contrasted to this is the complexity of the camera, particularly driven by the size of the telescope needed to collect enough photons.

Unlike many conventional satellite missions where the instantaneous observation is the primary measure and thus where SNR is a key component of the overall uncertainty budget, for climate benchmark applications this is not the case as the product of interest will always be temporally/spatially averaged to remove effects of localized anomalies. Although a GIFOV of $<250 \mathrm{~m}$ is desired for these applications, this is primarily to aid in assessing scene type and degree of cloud contamination. Most of the benchmark applications are concerned with scene aggregated observations over relatively large spatial and/or temporal scales. In these cases, effects of instrumental or observational noise, the dominant detrimental component of SNR, are effectively randomized and reduced significantly so that SNR makes little overall contribution to the uncertainty budget. Instead, instrument systematic effects and/or biases and drifts dominate the uncertainty achievable. Thus, the SNR for these applications can be $<50$ even at GIFOV of $250 \mathrm{~m}$. Similarly, for solar or lunar observations because the spacecraft and its sensors can effectively stare at its target and accumulate signal, the instantaneous SNR does not need to be demanding. For some climate applications e.g., ocean colour the SNR requirements can be more demanding i.e., $>1000$ but even here the spatial resolution can be relatively relaxed $>1 \mathrm{~km}$.

For applications where the instantaneous, spatially resolved measurement is of greater importance e.g., cross-calibration of a high-resolution land imager like Landsat or Sentinel 2 or imagery such as land use classification the radiometric uncertainty levels can be relaxed towards those of existing sensors but with the proviso that for the reference calibration process to be of value the reference sensor (TRUTHS) uncertainty should not dominate. Here, SNR will play a more significant role in the overall uncertainty budget and will likely be the ultimate limiter in terms of number of applications, when considering what is achievable for an affordable instrument design.

Table 2. Mission performance requirements (climate and calibration) red indicates a driving requirement. Signal to noise ratio (SNR) values are given with respect to an average Earth albedo of 0.3 and for the corresponding ground instantaneous field of view (GIFOV).

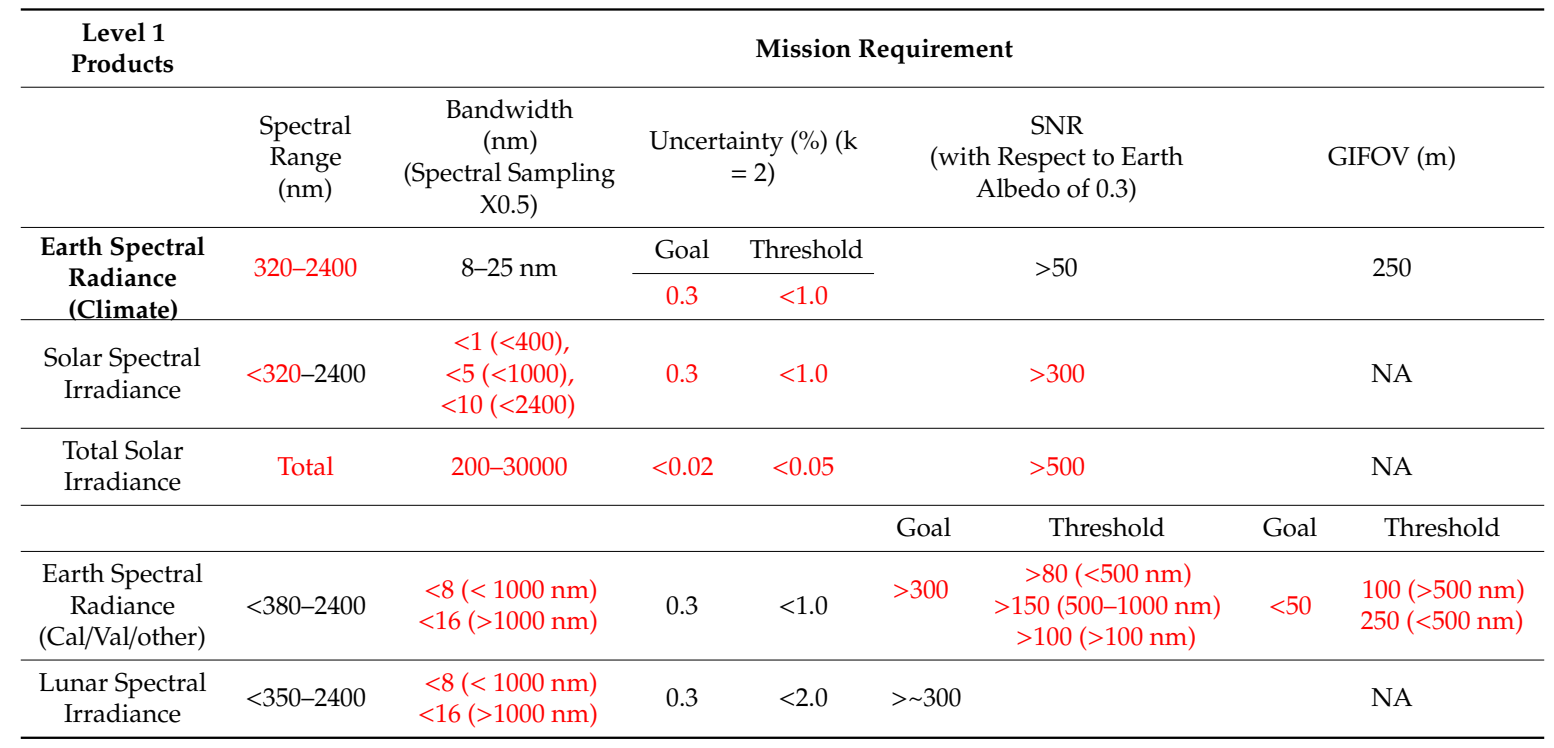

In terms of cross-calibration, the most demanding will be the ocean colour sensors like Sentinel-3 OLCI (Ocean and Land Colour Imager) and NASA PACE (Plankton, Aerosol, Cloud, ocean Ecosystem) missions, where overall uncertainty requirements at ToA is equivalent to a few tenths of a percent. At present, this effective uncertainty can only be approached as part of the overall retrieval process 
and requires the tuning of the sensor and algorithm to surface measurements made at one of two open ocean system vicarious calibration references, the buoys, MOBY and Bousolle.

TRUTHS, with an agile platform, can both spatially integrate and employ satellite motion compensation (rotating the satellite to compensate for its orbital movement to allow it to stare and temporally integrate over the target) to increase SNR for the specific measurement.

\subsection{Science User Requirements}

The operational and science user requirements are summarised here for the primary objectives, together with some example secondary objectives. With the development of the mission, towards launch and the increased engagement of the prospective user community, the breadth of potential identified secondary objectives is expected to increase significantly.

\subsubsection{Space Climate Observatory and Model Validation}

The most significant societal challenge facing mankind relates to the impact of climate change. Much of the uncertainty in climate forecasting results from the degree of modelled sensitivity of the climate response or 'feedbacks' to anthropogenic carbon emissions; the most significant being the modelled response of clouds, surface albedo, and aerosols. High-accuracy observations of perturbations in the energetic inputs and outputs to the climate system over decadal timescales are essential to extract the long-term climate trend from the ambiguity of natural variability, with each of the key components allowing detailed attribution analysis from its own unique spectral signature.

TRUTHS' observations provide a benchmark of the climate state from which such a trend may be determined by a subsequent mission of similar performance in a decadal timescale and provide a standard against which climate models can be tested and compared. Such testing is essential to improve confidence and reliability of future climate forecasts by reducing variance and uncertainty in the models [1]. Whilst confirming any trend requires a time-interval longer than a mission duration, the societal necessity of establishing an initial benchmark is thus an urgent priority. TRUTHS provides such a benchmark.

Observing both incoming and outgoing spectrally resolved solar radiation is a unique aspect of this mission and will improve our understanding of many processes where reflectance is a key parameter, including radiation budget and those requiring atmospheric correction.

Modern climate models directly use both the incoming and outgoing spectrally resolved solar radiation as constraints of the models' internal processes. Therefore, models can directly assimilate the TRUTHS products as a test of the models, with the spectrally resolved aspect allowing attribution of specific processes to the underlying model process [5]; not only verifying if the model produces an accurate overall radiative balance, but also ensuring the model is attributing the strength of the competing processes consistently. Essentially, it is not just about having a globally correct model, but that the model is correct for the right reasons.

The space climate observatory drivers for TRUTHS is long-term referencing of the climate change signal, enabling the observational measurement of the expected small trends (compared to their absolute reference value). The uncertainty requirements, that at first glance may seem excessively strict, are necessary to allow detection of these decadal level signals in as short a timescale as possible i.e., 'establish a reference with sufficiently small uncertainty that the signal from the accumulated trend can be reliably distinguished from it', and reflect those identified by GCOS following assessments from the science community.

The following two sub-sections relate to the incoming half of the radiation budget and in the second case its specific impact on the atmosphere.

\subsubsection{Total Solar Irradiance (TSI)}

The sun is of course the primary input energy source to the Earth and any, even very small change in its value will have significant impact on the Earth's climate. Fortunately, analysis of observation 
data [6] shows that the variability of the total solar irradiance (TSI) from minimum to the maximum in recent cycles of the solar activity does not exceed $0.1 \%$ or $\sim 1.4 \mathrm{~W} . \mathrm{m}^{-2}$, which translates into a direct radiative forcing of about $\sim 0.25 \mathrm{~W} . \mathrm{m}^{-2}$. However, a long-term proxy of solar activity, the solar modulation function, shows that in the past 50 years, solar activity was at a 500-year maximum, $[7,8]$, with intervening recent minima (Maunder (1645-1715) and the Dalton (1790-1830)), which had noticeable impact on the Earth's climate.

The past 30 years of satellite observations cannot be assumed to characterise the full range of possible changes of the solar irradiance over longer time scales. Although the magnitude of estimates of solar output have been downgraded in the IPCC report [4], there is considerable disagreement in the historical magnitude of the solar output variation and climate predictions into the future are best served with a continuous high accuracy, unambiguous record. GCOS require TSI with high precision and absolute uncertainty better than $0.01 \%(\mathrm{k}=1)$ is needed in the long-term (Table 1 ), and because it is impossible to confidently have a single instrument in orbit over the long-term immune of systematic change, traceability to SI is essential. The current extra-terrestrial instrument-based record not only is unable to meet this level of accuracy, it contains biases 10 times the magnitude of the solar cycle variation, which can only be partially reconciled, without SI traceability [9].

\subsubsection{Solar Spectral Irradiance (SSI)}

At the fundamental level, to understand the energy exchange within a system, both the incoming and outgoing energy needs to be understood. The current assessment is that solar variability has not been a major contributor to climate over the medium term (last 35 years).

Arguably of greater importance than the TSI is the variation of ultra-violet (UV) solar spectral irradiance below $\sim 400 \mathrm{~nm}$. This solar-cycle-varying UV contribution impacts atmospheric chemistry, thermal interface of the atmosphere with the oceans, the severity of El-Nino Southern Oscillation (ENSO), and is an early indicator of space weather events [10]. Yet, measurements of it are controversial with significant model output variation in the troposphere and stratosphere because of observational measurement uncertainty. Together with a question with the variation in near-IR SSI within the solar cycle [11], work is needed to reconcile the TSI and SSI measurements to date. The SI-traceable measurement of both TSI and SSI by TRUTHS will provide the much needed robustly anchored dataset needed to address these discrepancies.

For both the reflectance drivers, and that of the Earth-reflected radiance to be quantified to an accuracy level adequate to detect climate delta signals, the required accuracy on the SSI should match that of the Earth-reflected radiance. The GCOS requirement for SSI is $<0.3 \%$ from 200 to $2400 \mathrm{~nm}$ (see Table 1).

\subsubsection{Radiometric/Parameter Requirements: Climate Benchmark}

Table 3 provides a summary of the radiometric requirements of the mission's space climate observatory fiducial dataset derived from key applications.

The justification for the requirements is summarised below.

Spectral range-Ideally the entire solar reflective band from $<200 \mathrm{~nm}$ to $5000 \mathrm{~nm}$ would be measured, with all the relevant energy of interest; over $99.5 \%$ of the total reflected energy from the Earth to space is within the spectral range from $300 \mathrm{~nm}$ to $2400 \mathrm{~nm}$ under virtually all atmosphere and surface conditions, as discussed by the CLARREO team and illustrated in Figure 2. 45 contained in [12]. Therefore, in terms of total reflected radiation, from an imbalance perspective, measurements do not need to cover the entire spectrum but only the range in which sufficient reflected solar energy is enclosed. The minor correction from the uncovered spectral regions can be made using radiative transfer calculations as the impact of the additional uncertainty is negligible to the overall sum.

A summary of estimated error in total reflected solar energy is shown in Table 4 (reproduced here from CLARREO studies [12]) as a function of instrument spectral coverage globally and for selected scene types. 
Table 3. Mission requirements for climate benchmark observations of reflected solar radiance, and total and spectral solar irradiance. Spectral resolution is driven by the various climate variables. Accuracy is driven by the climate-benchmarking requirement to detect trends above natural variability.

\begin{tabular}{|c|c|c|c|c|}
\hline $\begin{array}{c}\text { Mission } \\
\text { Requirement }\end{array}$ & $\begin{array}{c}\text { Parameter: Proposed } \\
\text { Value }\end{array}$ & Driving Mission Objective & Required & Desired \\
\hline \multirow{6}{*}{$\begin{array}{c}\text { SI traceable } \\
\text { measurement of } \\
\text { the solar reflected } \\
\text { spectrum }\end{array}$} & \multirow{3}{*}{$\begin{array}{l}\text { Spectral range: } \\
320 \mathrm{~nm}-2400 \mathrm{~nm}\end{array}$} & $\begin{array}{l}\text { Nadir Reflectance Spectral } \\
\text { Climate Change Benchmarks }\end{array}$ & $<350 \mathrm{~nm}-2300 \mathrm{~nm}$ & \\
\hline & & Earth Radiation budget & & $\begin{array}{l}\text { From } 320 \mathrm{~nm} \text { to } \\
2400 \mathrm{~nm}\end{array}$ \\
\hline & & $\begin{array}{l}\text { Plant optical traits and } \\
\text { minerals }\end{array}$ & $380 \mathrm{~nm}-2400 \mathrm{~nm}$ & $\mathrm{Up}$ to $2500 \mathrm{~nm}$ \\
\hline & $\begin{array}{c}\text { Accuracy: } \\
0.3 \%(\mathrm{k}=2)\end{array}$ & $\begin{array}{l}\text { Trend estimation of cloud } \\
\text { feedback }\end{array}$ & $0.3 \%(\mathrm{k}=2)$ & \\
\hline & $\begin{array}{l}\text { Spectral resolution: } \\
1 \mathrm{~nm}-10 \mathrm{~nm}\end{array}$ & $\begin{array}{l}\text { Nadir Reflectance Spectral } \\
\text { Climate Change Benchmarks }\end{array}$ & $1 \mathrm{~nm}-10 \mathrm{~nm}$ & \\
\hline & $\begin{array}{c}50 \mathrm{~m} \text { (land) } 250 \mathrm{~m} \\
\text { (ocean) }\end{array}$ & Cloud masking & $<250 \mathrm{~m}$ & $<150 \mathrm{~m}$ \\
\hline \multirow{2}{*}{$\begin{array}{l}\text { SI traceable } \\
\text { measurement of } \\
\text { total solar } \\
\text { irradiance }\end{array}$} & $\begin{array}{c}\text { Spectral range: } 0.2->30 \\
\mu \mathrm{m}\end{array}$ & $\begin{array}{l}\text { Solar variability and Earth } \\
\text { Radiation Budget }\end{array}$ & $0.2->30 \mu \mathrm{m}$ & \\
\hline & $\begin{array}{c}\text { Accuracy: } \\
0.02 \%(\mathrm{k}=2)\end{array}$ & $\begin{array}{l}\text { Solar variability and Earth } \\
\text { Radiation Budget }\end{array}$ & $<0.02 \%(\mathrm{k}=2)$ & \\
\hline \multirow{2}{*}{$\begin{array}{l}\text { SI traceable } \\
\text { measurement of } \\
\text { solar spectral } \\
\text { irradiance }\end{array}$} & $\begin{array}{l}\text { Total Spectral range: } \\
320 \mathrm{~nm}-2400 \mathrm{~nm}\end{array}$ & Solar variability and ozone & $<350 \mathrm{~nm}-2400 \mathrm{~nm}$ & $200 \mathrm{~nm}-2400 \mathrm{~nm}$ \\
\hline & Accuracy: $0.3 \%(\mathrm{k}=2)$ & Solar variability & $0.3 \%(\mathrm{k}=2)$ & \\
\hline $\begin{array}{l}\text { Reference } \\
\text { calibrations }\end{array}$ & As for radiance above & $\begin{array}{l}\text { Reference Intercalibration: } \\
\text { particularly for ocean colour } \\
\text { monitoring sensors }\end{array}$ & $<400 \mathrm{~nm}->1000 \mathrm{~nm}$ & $200 \mathrm{~nm}-2400 \mathrm{~nm}$ \\
\hline
\end{tabular}

Table 4. Estimated error in total reflected solar energy.

\begin{tabular}{|c|c|c|c|c|}
\hline \multirow{2}{*}{ Scene Type } & \multicolumn{4}{|c|}{ Percent Error due to Limitation of Spectral Range } \\
\hline & $32-2300 \mathrm{~nm}$ & $320-2400 \mathrm{~nm}$ & $310-2300 \mathrm{~nm}$ & $310-2400 \mathrm{~nm}$ \\
\hline Global & $0.09 \%$ & $0.07 \%$ & $0.05 \%$ & $0.03 \%$ \\
\hline All-sky Ocean & $0.10 \%$ & $0.08 \%$ & $0.04 \%$ & $0.03 \%$ \\
\hline All-sky Land & $0.08 \%$ & $0.06 \%$ & $0.05 \%$ & $0.04 \%$ \\
\hline Clear Ocean & $0.16 \%$ & $0.15 \%$ & $0.05 \%$ & $0.04 \%$ \\
\hline Clear Desert & $0.10 \%$ & $0.07 \%$ & $0.07 \%$ & $0.04 \%$ \\
\hline
\end{tabular}

This leads to the goal requirement on spectral extent of $<320 \mathrm{~nm}-2400 \mathrm{~nm}$. The UV extent is particularly important for the solar spectral irradiance application where measurements to below the atmospheric ozone band at $290 \mathrm{~nm}$ would be ideal [13] but can be limited to $320 \mathrm{~nm}$ to avoid overly driving the mission specification, with the Earth-reflected radiance requirement of spectrally resolved data $>320 \mathrm{~nm}$.

Spectral Bandwidth - the Earth-reflected radiance spectral resolution is driven by the need to resolve the spectral signature of climate processes and is modest at $8 \mathrm{~nm}$ to $25 \mathrm{~nm}$ full width at half-maximum (FWHM). Improved information content is only gained with a spectral resolution $<3 \mathrm{~nm}$, where gas-phase processes start to be resolved. Cloud processes require $<10 \mathrm{~nm}$ spectral resolution so as not to degrade information content. However, the SSI measurement is the key driver for the climate space observatory driver, with ideally $<1 \mathrm{~nm}$ resolution below $400 \mathrm{~nm}$, and $5 \mathrm{~nm}$ to $10 \mathrm{~nm}$ resolution throughout the visible-Short Wave Infra-Red (SWIR) to allow models to adequately make use of the data. The latter Vis/SWIR bandwidths are also close to optimum for reference calibration requirements of most operational sensors, where the requirement is to enable adequate 
spectral matching of 'customer' sensor bands, and/or to enable spectral characterization of a spectrally smooth calibration target (e.g., desert, deep convective cloud, etc.) to allow interpolation for high spectral resolution sensors including those used for atmospheric chemistry related applications e.g., GHG sensors.

Radiometric accuracy-the $0.3 \%(\mathrm{k}=2)$ applied to an average global albedo of 0.3 , implies a 0.001 reflectance uncertainty requirement. The $0.3 \%(\mathrm{k}=2)$ spectrally integrated driver (needed to determine cloud radiative forcings and feedbacks), translates to a $0.3 \%$ to $1 \%(\mathrm{k}=2)$ spectrally resolved requirement, dependent on the wavelength $[14,15]$. The less strict requirements are in the strong absorption bands that contain less information content.

Signal to noise ratio (SNR) - the climate observatory native SNR goal requirement is 2300 in the vis-NIR, commensurate with the $0.3 \%(\mathrm{k}=2)$ accuracy requirement in the vis-NIR. However, as this is linked to IFOV, it should be noted that this instantaneous SNR level is not required for most of the earth viewing benchmark requirements, which will average over much larger spatial and/or temporal zones. This allows significant relaxation in SNR as the random component of the measurement is reduced. The instantaneous SNR requirement is higher for ocean colour and low signal water leaving applications, but this can be achieved with appropriate spectral and spatial binning and an increased dwell time attained from the platform agility.

Ground instantaneous field of view (GIFOV)— the space climate observatory GIFOV is driven by the need to identify and differentiate clouds within the dataset, with a requirement of $\sim 250 \mathrm{~m}$ spatial resolution [16] as a maximum. However, for cloud-free land applications, a higher $50 \mathrm{~m}$ GIFOV is valuable for many secondary applications involving land classification in some form and cross-calibration of higher resolution sensors.

Orbit-for climate benchmarking applications there is a preference (in the reflected solar spectral region) for measurements over a full diurnal cycle and therefore a precessing orbit of $90^{\circ}$, to minimise the sampling aliasing in the data record.

Pointing-The pointing requirement is split into two components:

- Nadir Earth-viewing pointing requirement for climate applications is only 1deg.

- Solar observations need a pointing accuracy of $<0.1 \mathrm{deg}$, maintained over one minute, to provide the TSI, allowing for the CSAR time constant with the proviso that the FoV is entirely within the solar disc, achieved with a simple quadrant detector on the instrument front-plate, as flown in heritage Physikalisch-Meteorologisches Observatorium Davos (PMOD) missions.

\subsubsection{Climate Change Services}

A secondary objective for TRUTHS' space climate observatory data is to improve the accuracy and usefulness of downstream value-added services, expediting the European Commission's goal of leading the development of the climate services market [17] and contributing significantly to the quality of data feeding into the Copernicus Climate Change Service (C3S).

The broad range of benefits and long timescales needed to detect trends reliably and to establish robust climate data records (CDRs) highlights the value of an early launch. Delaying the creation of such a system extends the time needed to establish reliable climate records and creates the constant risk of time-series being lost completely should an adequate overlap of missions be missed (due to lack of robust traceability/stability of current sensors). The 'value of information' that climate observing systems such as TRUTHS provide was modelled at trillions of dollars due to damages avoided from better-informed decision making [18]. TRUTHS is proposed as a minimum five-year mission, which is adequate to establish the key climate and calibration benchmarks and demonstrate the benefits at cost-compliance, although a longer duration of eight or more years is targeted.

Although the technical specification of TRUTHS is unlikely to be driven by the needs of any one specific ECV, it is helpful to consider where the mission can play a valuable role if only to ensure that potential small modifications are appropriately evaluated. Our current selection of candidate ECVs is based on the following criteria: (1) Importance to global-scale climate change and (2) accessible to 
the technical scope of the mission. Table 5 provides a summary of the main ECVs, key elements of the primary societal benefit area (climate) addressed by TRUTHS by its own observations or through reference calibration of other sensors. The fiducial nature, and, the broad spectral/spatial coverage of the mission ensures it also provides significant impact to food and water, natural resources and energy, and disasters, health and pollution. The solar reflective domain is a rich source of information and the hyperspectral capabilities of TRUTHS, together with its high accuracy allows the various signatures to be extracted and de-coupled.

Table 5. A summary of climate change variables where TRUTHS can have significant impact either directly or via reference inter-calibration. The selection of variables is based on the following criteria: (1) Importance to global-scale climate change, (2) availability of satellite-based climate data records, and (3) measurability from passive sensors in the spectral range of TRUTHS i.e., near UV to SWIR.

\begin{tabular}{|c|c|c|c|}
\hline Climate Variable & Role & $\begin{array}{l}\text { TRUTHS Providing } \\
\text { Direct Observation }\end{array}$ & $\begin{array}{l}\text { TRUTHS Providing } \\
\text { Reference Calibration }\end{array}$ \\
\hline Solar Irradiance & Climate forcing & yes & yes \\
\hline Earth Radiation Budget & Climate forcing, feedback & yes & yes \\
\hline Surface Albedo & Albedo feedback & yes & yes \\
\hline Cloud Cover & Cloud feedback & yes & yes \\
\hline $\begin{array}{l}\text { Cloud Particle Size } \\
\text { Distribution }\end{array}$ & & $\begin{array}{c}\text { yes, through spectral } \\
\text { benchmarking }\end{array}$ & yes \\
\hline Cloud Effective Particle Size & & & yes \\
\hline Cloud Ice/Water Content & & & yes \\
\hline Cloud Optical Thickness & & & yes \\
\hline Water Vapour & Column water vapour response & yes & yes \\
\hline Ozone & Stratospheric ozone feedback & $\begin{array}{l}\text { no (limited spectral } \\
\text { resolution) }\end{array}$ & yes \\
\hline Aerosols Optical Depth & $\begin{array}{c}\text { Climate forcing } \\
\text { Atmospheric correction }\end{array}$ & $\begin{array}{c}\text { (limited temporal/ } \\
\text { spatial coverage) } \\
\text { yes }\end{array}$ & $\begin{array}{l}\text { yes } \\
\text { yes }\end{array}$ \\
\hline Ocean \& Lake Colour & Carbon cycle & yes & yes \\
\hline Ice and Snow Cover & Albedo feedback & yes & yes \\
\hline Vegetation Indices & $\begin{array}{c}\text { Carbon Cycle and Albedo } \\
\text { feedback }\end{array}$ & yes & yes \\
\hline Land Cover/Land Use & Surface Radiative Forcing & yes & yes \\
\hline GHGs & $\mathrm{CO}_{2} / \mathrm{CH}_{4}$ emissions monitoring & $\begin{array}{l}\text { Yes but only large } \\
\text { scale emitters }\end{array}$ & Yes \\
\hline
\end{tabular}

Commercial end-users have been consulted to understand the potential economic value of TRUTHS' data and its impact on the EO observing system as a whole, with globally significant insurance companies commenting that TRUTHS could 'underpin robust and credible products used across the industry'.

\subsubsection{Reference Calibration of Sensors-Test-Site Characterisation}

The satellite community has sought to establish well-characterised 'test-sites' and associated methods of use, ideally instrumented at ground level, to provide a means of both calibration and validation of level 1 radiances and, in some cases, level 2 or higher products.

In recent years, significant efforts have been made by the international community (through CEOS and GSICS) to consolidate and focus efforts on a sub-set of these sites/methods to aid sensor inter-operability and harmonisation. Efforts have started to establish true calibration networks at these 
'CEOS reference standard test sites', analogous to the long established AERONET sites for aerosols, together with developing the tools needed to facilitate cross-calibration between sensors at these sites.

- The RadCalNet instrumented land network [19,20];

- Non-instrumented stable (usually desert, but also snowfields) land sites known generically as Pseudo-Invariant Calibration Sites (PICS) [21];

- Ocean buoys at Moby and Bousolle [22];

- $\quad$ The Aeronet-OC network;

- Extra-terrestrially, the Moon is a commonly used reference site [23].

In addition, there are the use of Rayleigh scattering over the oceans, deep convective clouds, and Simultaneous Nadir Observation over any of the above or indeed any other suitable location on the Earth.

Calibration using PICS has historically provided highly reproducible calibrations, when suitably averaged to remove unknown effects due to atmosphere, spectral, and residual uncorrected surface Bi-directional Reflectance Factor (BRF) but are limited in terms of absolute accuracy through lack of any reference measurement.

There is a fundamental need for accurate hyperspectral referencing, provided by TRUTHS, as a means to make a huge step change in long-term performance characterisation. Even with a minimal improvement in uncertainty but with high confidence level in its SI traceability, an anchoring reference would allow harmonisation and certainty in the true reference value.

Current cross-comparison strategies assume the site surface reflectance is stable (reasonable and proven for some sites) and by taking multiple samples, variables like solar/sensor view (after correction) and atmosphere are sufficiently randomised. Chander et al. [24] provides an analysis of what can be achieved for a range of sensor types using Landsat and MODIS as examples. This study concludes that for all geometric/positioning sources of error in the cross-comparison process, the uncertainties are $<\sim 0.3 \%$ confirming the analysis summarised below in Table 6 by Gorono et al [25], in the worst case for one spectral band a miss-registration error (lack of knowledge) of three pixels contributed $<0.5 \%$ uncertainty. The only significant source of uncertainty in their analysis stems from the prospect of unknown spectral change in the sensors band pass due to ageing of the filter. In their analysis, they assumed relatively large shifts of $\pm 5 \mathrm{~nm}$, which in turn led to uncertainties of up to $2.4 \%$. Such shifts may occur in practice on any sensor. However, they would be considered relatively extreme for a well-designed sensor and consequently such potential effects are in general not accounted for in the uncertainty budgets of sensors like Sentinel 2. It could therefore be misleading to consider this the limiting aspect of the uncertainty achievable in using such test-sites. Therefore, at present, this potential source of error is not included in the uncertainty budget attributed to the use of TRUTHS for cross-calibration. It may be, however, that cross-calibration with TRUTHS over a range of terrain types, with different spectral properties, may be able to assess if such changes are occurring, but this analysis has not as yet been undertaken.

TRUTHS will use a similar philosophy for the PICS site characterisation but will calibrate to an absolute reference, so determining the bias of each sensor from the actual reflectance, resulting in reliable harmonisation of the data record from multiple sensors. This SI-traceable anchoring of the true reference reflectance can not only be applied to current sensors but also to historical and future PICS site companions, long after the TRUTHS sensor mission life. The agility of TRUTHS would also allow the surface/ToA BRF of the PICS sites to be evaluated leading to improved corrections of seasonal variability.

The requirement drivers from this activity do not exceed that given in Table 2 as the overall uncertainty will be limited by the site BRF knowledge and atmospheric correction contribution i.e., the knowledge of the atmospheric state at the time of both the reference and target sensor overpass. 


\subsubsection{Calibration of sensors-SNO at PICS}

An enhancement to purely characterising the PICS sites comes from simultaneous nadir observations (SNO) viewing the nominally spatially uniform site. Although the discussion here focusses on a PICS site, the methodology is equally valid when using a deep convective cloud, a snowfield, or other site of sufficient size and characteristics for the field of view of the sensor under test and that of TRUTHS. The orbit and agility of TRUTHS provides additional opportunity for coincident $(<30 \mathrm{~min}$ ) view-matched sampling of a range of target sites, greatly reducing the number of samples needed to effectively randomise the unmatched residuals of associated contributors; with only a few matched overpasses needed to improve the absolute accuracy of a specific sensor (subject to respective SNR values), achievable on a relatively short timescale. Figure 2 shows the matched SNO between Sentinel 2 and TRUTHS assuming a nominal Swath of $50 \mathrm{~km}$ for a time window of $30 \mathrm{~min}$ and for 1 year. The exact number of crossovers (pixel/scene) over good quality targets and/or PICS needs to be calculated.

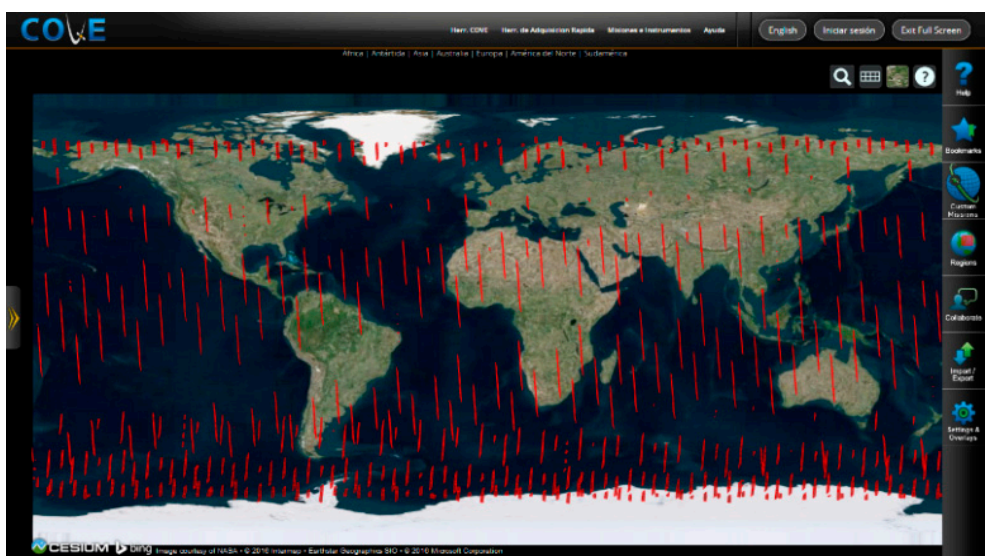

Figure 2. Multi-Spectral Imager (MSI) of Sentinel 2 and TRUTHS simulated overpasses for a one-year period with time interval of $30 \mathrm{~min}$ at nadir.

Table 6 provides the uncertainty budget from a study analysing the radiometric calibration upgrade of Sentinel 2 achievable using the Libya 4 PICS and TRUTHS system performance (Table 2) as a calibration reference. The details of the underpinning analysis can be found in [25]. This analysis accounts for spatial and spectral mismatch, solar and view angle variations, as well as atmospheric effects. The analysis concludes that for most bands a single coincident overpass of the two satellites enables a Sentinel 2 Multi-Spectral Imager (MSI) uncertainty upgrade in radiometric performance to $\sim 0.5 \%(\mathrm{k}=1)$. This assumes, as indicated in the MSI uncertainty budget, that the dominant uncertainty component is 'in-flight, radiometric calibration.

Similar analysis can be done for other sensors. Of course, to extract optimum value from such an in-flight calibration strategy there needs to be a good level of pre-flight characterisation of the sensor under test as this approach primarily only addresses radiometric gain/bias. However, through use of multiple sites it can also assess linearity, and some aspects of spectral performance although not necessarily fully correct for any errors that may be observed. This is not really a limitation as it reflects current practices-and addresses the principle uncertainty component listed in most sensor budgets. It is however true that with a reference sensor of the quality of TRUTHS in-orbit, future sensors may take account of this and ensure that design and pre-flight characterisation will be optimised to fully utilise the benefits.

A summary of the impact of TRUTHS' key performance parameters on the cross-calibration of a sensor like Sentinel-2 is given in Table 6 and below is a discussion on considerations needed for the specification of TRUTHS (Tables 2 and 7). 
Table 6. Uncertainty budget $(k=1)$ achievable for the upgrade of the radiometric performance of Sentinel 2 using TRUTHS as a reference sensor and the Libya-4 PICS. The contributions marked with an asterisk are included in the total at the bottom of the table. Some contributions are calculated based on a potential range of conditions and only one variant is used in the total.

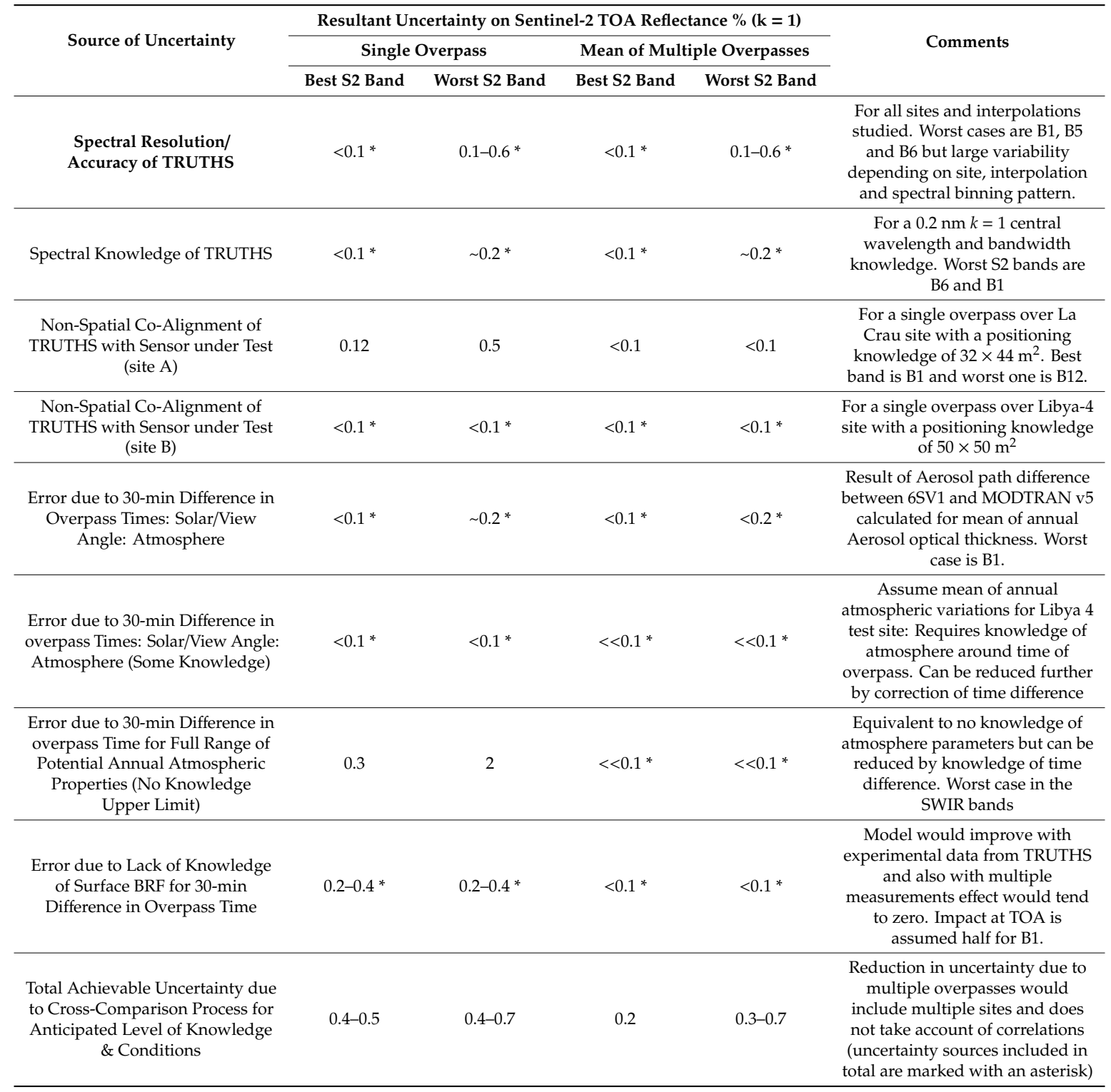

\subsubsection{TRUTHS specifications resulting from reference calibration using test-sites}

The following discussion summarises the mission requirements resulting from the needs of the reference calibration objective and where appropriate contrasts them with those needed for climate.

Spectral range - the spectral range should cover that of the sensors being cross-calibrated. This is less strict than the Space climate observatory spectral requirement and could be limited to $\sim 380 / 400$ to $2400 \mathrm{~nm}$.

Spectral Bandwidth - the Earth-reflected radiance spectral resolution is driven by the need to match the spectral resolution of the sensors under test $[3,12]$ with an acceptable residual uncertainty in the spectral matching. The spectral bandwidth (spectral sampling interval is half the bandwidth) requirement of $8 \mathrm{~nm}<1000 \mathrm{~nm}$ and $16 \mathrm{~nm}>1000 \mathrm{~nm}$ allows this spectral matching for the band type sensors (non-spectrometer) and is of a similar magnitude to the new generation of hyperspectral 
imaging spectrometers. This is consummate with those needed for cloud processes identified for the space climate observatory requirement.

Radiometric accuracy - the reference sensor radiometric accuracy should be considered alongside the other uncertainties in matching the inter-calibration signal. These other considerations are typically of the $0.5 \%(\mathrm{k}=2)$ magnitude. The reference sensor radiometric uncertainty is the main budget contributor for values above $0.5 \%(\mathrm{k}=2)$ but values $\leq 1 \%(\mathrm{k}=2)$ are acceptable for most sensors studied. The SCO requirement is the driving requirement on the radiometric accuracy.

Signal to noise ratio (SNR) - the SNR requirements is convolved with other factors including the sampling regime, number of samples, and the spectral and spatial binning needed to match the sensor under-test. Generally, the SNR requirement is related to the GIFOV, brightness of target, and/or number of samples and radiometric accuracy requirements $\sim 300$ in the visible reflects the goal uncertainty so that it is not limiting for a direct instantaneous observation. For specific ocean colour applications, this requirement rises to $>1000$ and ideally 2000 in the blue, but as the observational pixel size for most Ocean Colour applications is $>1 \mathrm{~km}$, this higher SNR can be achieved with appropriate spectral and spatial binning and an increased dwell time attained from the platform agility. As a threshold, values commensurate with those of other sensors e.g., Sentinel 2, accounting for spectral bandwidth differences etc., are chosen so that SNR of TRUTHS does not limit the calibration or corresponding application. This requirement is more demanding than that of the space climate observatory and drives the mission design. However, achieving at least threshold should not present significant challenges.

Ground instantaneous field of view (GIFOV)—-the cross-calibration GIFOV is driven by the need to match the spatial resolution of the sensors under calibration, together with optimising the use of the L1 data for land surface applications. The goal GIFOV requirement is $50 \mathrm{~m}$, however there is some advantage of reducing this to $30 \mathrm{~m}$ (match other hyperspectral sensors) if detectors arrays do not compromise the overall swath significantly. This requirement is stricter for GIFOV than the space climate observatory.

Orbit-A precessing asynchronous orbit of $90^{\circ}$ facilitates a maximal number of SNO with other sensors for reference calibration. It should be noted that a high number of SNO' (for the reference calibration application) can also be achieved with other asynchronous orbits. The orbit altitude ideally needs to be $>100 \mathrm{~km}$ separated from the target sensor, ideally $>\sim 200 \mathrm{~km}$, to allow adequate time at the orbit crossing to match the views and increase overlap time for reference calibration. Sentinel $2 / 3$ are at $\sim 800 \mathrm{~km}$ altitude, with LandSat 8 around $700 \mathrm{~km}$ altitude. Too low an orbit increases drag and fuel lifetime and radiation damage. The baseline has at this stage been chosen to be optimal as a precessing polar orbit $\left(90^{\circ}\right)$ at a height of $609 \mathrm{~km}$ with a repeat time of 61 days.

Pointing-The pointing requirement for cross-calibration application is to match the partner sensor FoV, with an accuracy of $\sim<0.1 \mathrm{deg}$, and knowledge typically $<2 \mathrm{x}$ the GIFOV, so $100 \mathrm{~m}$ ( $\leq 1 \mathrm{arcmin}$ ). However, this is no more demanding than the navigation pointing knowledge. For special sites of interest, there is the ability to motion compensate and take multiple angle views and also take account of ground control points.

Observational plan-from a mission perspective, all that is additional in terms of specification is that regular observation of key CEOS test-sites should be built into the acquisition program. Calibration of the instrumented sites allows these sites to provide improved calibrations on timescales independent of intervals between TRUTHS overpass and that of the sensor under calibration. TRUTHS will target these CEOS endorsed sites providing high accuracy TOA spectrally, spatially, and angularly resolved radiances to maximise their potential usage and ensure SI traceability.

\subsubsection{Calibration of Sensors-Global SNOs}

An alternative approach to calibration of sensors using test-sites, is to use the matched, SNO overpasses over a wide variety of surface (and cloud-top) types. This strategy will provide many more matchups over a shorter time, spanning the full dynamic range of signal levels. The uncertainty budget will mirror that given in Table 6, with a slightly increased uncertainty for the BRF terms over less stable 
surfaces, in-homogeneity, and potentially the selection of the 'no knowledge' upper limit assumption on atmospheric correction variation within the temporal mismatch. However, as this approach takes advantage of the increase in matchups afforded by the increase in coincident occurrences, the mean of multiple overpasses budget is the valid one in this scenario.

The requirement drivers from this activity do not exceed that given in Table 2 and are commensurate with those summarised in Section 2.4.8.

\subsubsection{Calibration of Ocean Colour Sensors}

The WMO [26,27] and GCOS [4] requirement for water leaving radiance (ocean colour) is 'better than $5 \%(\mathrm{k}=2)^{\prime}$. Although there is some variation with wavelength and water type, in the blue-green, this translates to an absolute radiometric accuracy at ToA of $\sim 0.5 \%(\mathrm{k}=2)$. This scaling results from the water leaving signal being only $10 \%$ of the total observed by the satellite, the dominant signal coming from the atmosphere. TRUTHS incorporates this requirement in Table 2, although it should be noted that with threshold values this may only be achieved through reference calibration of other sensors, although with goal values for SNR this may also be globally achievable by TRUTHS, but the temporal coverage may not be adequate for some applications. For existing sensors, this resultant high accuracy is only achievable in an indirect manner through a process known as 'system vicarious calibration' using ocean buoys fitted with well-calibrated radiometers at very specific locations namely, MOBY and Boussole. TRUTHS enables sensor performance to be calibrated directly and algorithms to be tuned over a range of locations and water types. The TRUTHS SNR and GIFOV also allow analysis in coastal zones and inland waters that have previously not been readily accessible.

\subsubsection{Calibration for Greenhouse Gas Source and Sink Monitoring}

Although the spectral resolution of TRUTHS is not sufficient to directly derive gaseous-phase products, except for relatively large-scale emitters, the transfer of radiometric accuracy through cross-calibration does provide some significant benefit for the GHG community. To meet the goals of the Paris Accord of 2015, an international GHG virtual constellation is required, which will fundamentally require harmonisation of the different sensors, which must start from the level 1 radiometry. For example, NASA Jet Propulsion Laboratory (JPL) have performed a series of studies to characterize the impact of uncertainties in the absolute radiometric calibration on the XCO2 retrievals from the Orbiting Carbon Observatory-2 (OCO-2) sensor. The study showed the retrieval to be most sensitive to the absolute radiometric accuracy in the $\mathrm{O}_{2} \mathrm{~A}$-band channel. In that channel, a $1 \%$ error in the absolute radiometric calibration introduces a $\sim 0.1 \mathrm{ppm}(0.025 \%)$ error in XCO2 for an aerosol optical depth of 0.1 and a surface albedo of $\sim 0.1$, with the error increasing with increasing aerosol optical depth and surface albedo values. Principally, absolute radiometric errors can therefore manifest as spatially dependent biases while also contributing to the random error in the XCO2 soundings.

Similar impacts would be expected in other GHG missions, with the value of a unified absolute calibration across missions best demonstrated in any international effort to inter-operate sensors as part of a future international constellation. Section 2.4.6 d and Section 2.4.7 demonstrates the ability to cross-calibrate partner sensors to the $<1 \%$ level, allowing TRUTHS to limit the bias-related drift in GHG retrievals to the $<0.1 \mathrm{ppm}$ level.

\subsubsection{Lunar Calibration}

The moon is a unique calibration test-site. As an extra-terrestrial site (with no atmosphere to characterise between sensor and target) it has been shown to be radiometrically stable, after taking account of libration etc., allowing the stability of a satellite sensor to be monitored in orbit and inter-compared.

The dominant work in this field to date has been carried out by the United States Geological Survey (USGS) and its programme on the Robotic Lunar Observatory (ROLO) [28]. This has now been incorporated into an international program of the WMO GSICS with the creation of GIRO 
(GSICS Implementation of ROLO) as an international reference model. However, the current absolute radiometric accuracy of the moon $\sim 1 \%$ in terms of relative accuracy, but $5-10 \%$ in absolute radiometric terms [23], is not sufficient to allow it to be used as an absolute standard at climate quality levels. The requirement is to reduce the absolute uncertainty to $\sim<1 \%$ to allow the moon to be used a long-term validation site.

TRUTHS will radiometrically calibrate the moon allowing it to be used as a true SI transfer standard. This only requires that the platform regularly views the moon with its HIS at a range of libration conditions to build up a correction database for the GIRO model. This early achievement from the mission, the L1 lunar spectral irradiance data product, would allow historic missions to be re-calibrated and Fundamental Climate Data Records (FCDRs) to be extended backwards in time with greater accuracy, since many sensors have been observing the moon.

\subsubsection{Calibration to Support Atmospheric Correction}

For most geophysical applications, knowledge of the state of the atmospheric column-aerosols and water vapour content in particular-are critical to enable high accuracy retrievals over land and oceans, as well as for cross-calibration. TRUTHS will have the additional capability through its hyperspectral measurements to determine Aerosol optical depth and water vapour in a similar but enhanced manner to OLCI through its additional SWIR bands, and for dedicated targets the agile platform can also allow multiple angle observations to add additional constraints. This will not only provide some measure of the atmosphere for direct L1 dataset applications but will subsequently allow existing atmospheric codes to be tested, constrained, and improved, under a range of conditions. In addition, it will facilitate testing of other aerosol measuring networks for example the sensors of AERONET itself can be constrained by the improved accuracy of TRUTHS. It should also be noted that the SI-traceable ground measurements of RadCalNet, [19] linked to the SI-traceable ToA measurements of TRUTHS, allow rigorous validation of atmospheric correction over such sites.

\subsubsection{Vegetation Indices}

The TRUTHS spectral range covers a great number of vegetation indices at a spectral resolution capable of providing direct products. However, the orbit period and coverage would limit the usefulness of any such product to an instantaneous map or long-term monitoring. The continuous hyperspectral coverage and high accuracy would however be useful in algorithm development and as a testbed for other missions.

The benefit is the absolute cross-calibration and the magnitude of the decadal-scale climate change signals expected in these indices. For example, a typical normalised difference vegetation index (NDVI) value of 0.3 , against which a $1 \%$ calibration accuracy may not seem significant. However, the change in NDVI might be less than 0.005 per year over 10 years i.e., less than 0.05 in a decade. Absolute uncertainties of the order of $1 \%$ are a significant contribution over these longer timeframes. One of the limiting factors in vegetation indices products are uncertainties in the atmospheric correction, and knowledge of the state of the atmosphere used to provide bottom of the atmosphere (BoA) radiances.

\section{Mission Architecture}

This section briefly describes the key components of the mission architecture, which have evolved and simplified since the mission was first conceived in $2001[29,30]$.

\subsection{System Requirements}

The TRUTHS mission requirements are summarised in Table 7. 
Table 7. TRUTHS mission requirements.

\begin{tabular}{|c|c|}
\hline Mission Requirement & Parameter \\
\hline $\begin{array}{l}\text { Spectral coverage (Earth and Sun) } \\
\text { (spectrometer) }\end{array}$ & $\begin{array}{l}320-2400 \mathrm{~nm} \text { (potential relaxation of spectral range if technology drivers } \\
\text { are too great but would limit value at shorter band for solar applications) } \\
\text { with continuous coverage. }\end{array}$ \\
\hline Radiometric Accuracy (spectrometer) & $\begin{array}{l}\text { Spectrally integrated reflectance product - } 0.3 \%(\mathrm{k}=2) \text { on the reflectance } \\
\text { of } 0.3\end{array}$ \\
\hline Radiometric Accuracy & $\begin{array}{l}\text { Spectrally resolved reflectance product }-0.3 \% \text { for the key spectral regions, } \\
\text { extending to } 0.5-1.0 \% \text { in less sensitive or energetically low-contributing } \\
\text { spectral positions. }\end{array}$ \\
\hline $\begin{array}{l}\text { Radiometric Accuracy (total combined } \\
\text { uncertainty) }\end{array}$ & $\begin{array}{c}\text { Spectrally integrated ( } 0.32 \text { to } 2.4 \mu \mathrm{m}) \text { Earth reflected solar radiance } \\
\text { product } 0.3 \%(\mathrm{k}=2)\end{array}$ \\
\hline Radiometric Accuracy & Spectrally resolved $(0.32$ to $2.4 \mu \mathrm{m})$ solar irradiance $0.3 \%(\mathrm{k}=2)$ \\
\hline Radiometric Accuracy (TSI) & $0.2 \mu \mathrm{m}$ to $30 \mathrm{um}$ integrated total irradiance $0.02 \%$ accuracy $(\mathrm{k}=2)$ \\
\hline Spectral Resolved Spectral Range & 320 to $2400 \mathrm{~nm}$ continuous \\
\hline Spectral Resolution & $\begin{array}{l}\text { For reference calibration need to match customer sensor: } 8 \mathrm{~nm} \text { bandwidth } \\
\sim 4 \mathrm{~nm} \text { steps) Can be relaxed to } 25 \mathrm{~nm} \text { for some non-critical wavelength } \\
\text { regions. }\end{array}$ \\
\hline Signal to Noise Ratio & $\begin{array}{l}\text { Adequate to meet accuracy levels in products with appropriate averaging. } \\
\text { Reference calibration and level } 2 \text { retrievals require }>100 \text { and ideally }>300 \\
\text { for at least visible spectral region at highest resolution }\end{array}$ \\
\hline Instantaneous Spatial Resolution (IFOV) & $<250 \mathrm{~m}$. ideally $30-50 \mathrm{~m}$ \\
\hline Polarisation Sensitivity & $\begin{array}{l}0.5 \%(\mathrm{k}=2) \text { for } \lambda<1000 \mathrm{~nm} \\
0.75 \%(\mathrm{k}=2) \text { for } \lambda>1000 \mathrm{~nm} \text { (Although for many applications these can be } \\
\text { relaxed. They are primarily to support off-axis reference calibrations of } \\
\text { other sensors and associated atmospheric corrections). }\end{array}$ \\
\hline Orbit & polar 90-degree inclination with altitude of $609 \mathrm{~km}( \pm 200 \mathrm{~m})$ \\
\hline $\begin{array}{l}\text { Reference inter comparison samples (large } \\
\text { FOV climate focused sensors) }\end{array}$ & $\begin{array}{l}2000 \text { samples (assuming SNR }<50 \text { will reduce with improved SNR) within } \\
1 \text { deg and } \pm 5 \text { min. (this assumes non selected targets for calibration). For } \\
\text { higher GIFOV sensors and higher SNR using SNO this value can reduce } \\
\text { significantly to }<10\end{array}$ \\
\hline Continuity of operation & $\begin{array}{l}\text { Sufficiently continuous to not increase the sampling bias error } \\
\text { contribution to represent a significant uncertainty contribution. }\end{array}$ \\
\hline Revisit & $\begin{array}{c}\text { As frequent as possible but }<61 \text {-day revisit as determined by the } 90 \mathrm{deg} \\
\text { polar orbit. }\end{array}$ \\
\hline Coverage & Global nadir spectral radiances \\
\hline Sampling time & Continuous during sunlit phase of the Earth \\
\hline Swath & 50 to $100 \mathrm{~km}$ minimum \\
\hline Quantisation & 14 bit \\
\hline Pointing Accuracy & $<1 \mathrm{~km}$ \\
\hline Pointing Knowledge & $<3 \times$ GIFOV $(<150 \mathrm{~m})$ Goal of $50 \mathrm{~m}$. \\
\hline
\end{tabular}

The above mission requirements in Table 7 are achieved through:

(a) The payload instrument: A hyperspectral imaging spectrometer (HIS), which can be used to measure the Earth, Sun, and Moon.

(b) The radiometric calibration of that HIS directly, regularly, and traceably to an on-board SI primary standard.

(c) The choice of an orbit that supports climate benchmark measurements and increases the number of simultaneous cross-calibrations.

(d) The use of a small, agile platform. 


\subsection{Payload}

\subsubsection{Overview}

The main observational instrument in TRUTHS is a hyperspectral imaging spectrometer (HIS), with continuous spectral sampling extending across the UV-VIS-SWIR ( $320 \mathrm{~nm}$ to $2400 \mathrm{~nm}$ ) and 50-100 m GIFOV, not dissimilar in headline capability to the DLR EnMap, NASA Hyperion and HyspIRI, ASI PRISMA or the future Copernicus Chime. Although as a baseline, TRUTHS' HIS is able to employ a much smaller telescope aperture of $\sim 50 \mathrm{~mm}$ diameter compared to the $200 \mathrm{~mm}$ aperture required by EnMap, as TRUTHS is not seeking to address all the same goals as these sensors, for instance high instantaneous SNR at $30 \mathrm{~m}$ GIFOV.

The disruptive innovation of TRUTHS stems from incorporating a space-adapted primary SI-standard onto the satellite bus and its use with a novel on-board calibration system. This mimics the method used terrestrially at NMIs, allowing TRUTHS to provide hyperspectral data immune from drift or biases for the lifetime of the mission. The method, which will be described below, enables TRUTHS to meet the performance requirements summarised in Tables 2 and 7, which are needed to address the mission objectives and science applications outlined in Section 2.

Table 2 shows the L1 product mission requirements that need to be achieved from the TRUTHS payload. The goal uncertainties specified, whilst challenging and unprecedented, are achievable using the disruptive on-board calibration system. The goal values for all the parameters would ensure that maximum science benefit is achieved, however some relaxation can be allowed towards a threshold value (close to that of the best current/planned sensors) and still achieve significant value, particularly from its cross-calibration function of the mission. However, from a climate perspective, the decrease in science benefit is non-linear in relation to uncertainty relaxation.

A summary of the payload instruments is provided below together with an overview of the on-board calibration method. It should be noted that the relatively unique asynchronous orbit of TRUTHS, whilst providing many observational benefits presents significant additional challenge to both the spacecraft and payload. Varying thermal and solar viewing (power) conditions add complexity to the mission. Thus, as part of the pre-flight calibration/characterisation and during the commissioning phase, calibrations of the HIS will be performed under a variety of simulated/real orbit conditions to assess any variances due to inadequate thermal stabilities.

\subsubsection{Hyperspectral Imaging Spectrometer (HIS)}

The primary measurand of TRUTHS is spectrally resolved incoming and Earth-reflected solar radiation. The spectral range, resolution, and accuracy are key boundary conditions, which ultimately limit the range of applications of the dataset (see Tables 3 and 5 and discussion in Section 2.4).

The HIS, through a novel approach, can be used both to image the Earth, measuring its spectral radiance, the Moon, measuring its spectral irradiance, and also to measure incident solar spectral irradiance. This concept not only minimises the instrumentation needed, but also allows us to derive ToA reflectance with the added confidence of exact spectral matching, through use of a common spectrometer. It also has the advantage that solar features can provide additional anchor points for spectral calibration. As an illustration of the viability the details of one indicative implementation of the HIS, with its specification, is given in Table 8.

Designs for the HIS have been produced by several organisations and Figure 3 shows a version from SSTL (UK). However, this is indicative and not necessarily the final design that will be implemented following the Phase AB1 studies. 
Table 8. Indicative hyperspectral imaging spectrometer (HIS) specifications based on an indicative implementation.

\begin{tabular}{|c|c|c|}
\hline Parameter & Value & Comment \\
\hline Spectral bands & $\begin{array}{l}320 \mathrm{~nm}-400 \mathrm{~nm} \\
400 \mathrm{~nm}-2450 \mathrm{~nm}\end{array}$ & $\begin{array}{l}\text { Assumes use of a wide spectral band } \mathrm{HgCdTe} \\
\text { detector and a separate Si detector through a } \\
\text { common telescope with a prism based spectrometer }\end{array}$ \\
\hline Swath & $100 \mathrm{~km}$ & $\begin{array}{c}\text { Corresponding GIFOV will depend on choice of } \\
\text { detector (number of pixels) } 2000 \text { and } 3000 \text { options are } \\
\text { now readily available }\end{array}$ \\
\hline Spatial Resolution & 50-100 m GIFOV & Depends on detector and SNR \\
\hline Spectral Sampling & $\begin{array}{l}4 \mathrm{~nm}<1000 \mathrm{~nm} \\
8 \mathrm{~nm}>1000 \mathrm{~nm}\end{array}$ & $\begin{array}{l}\text { UV native spectral resolution } \sim 1 \mathrm{~nm} \text {, with bandwidth } \\
\text { typically } 2 X \text { this value }\end{array}$ \\
\hline SNR & $>150$ & $\begin{array}{l}\text { Climate benchmarking only require } \sim 50 \text { due to zonal } \\
\text { averaging but higher values required for } \\
\text { cross-calibration and secondary applications }\end{array}$ \\
\hline Detector Technology & $\begin{array}{l}\text { UV: Si CMOS } \\
\text { VIS-SWIR: MCT }\end{array}$ & $\begin{array}{c}\text { Teledyne e2v CIS115/CIS120 (250K) } \\
\text { Teledyne e2v CHROMA-D 1/2/3k x } 512 \text { (135K) }\end{array}$ \\
\hline Imager Configuration & Refractive (prism) & \\
\hline Telescope & 1 telecentric $5 \mathrm{Al}$ mirror telescope & One common entrance pupil for calibration. \\
\hline Entrance Pupil & $45 \mathrm{~mm}$ diameter & \\
\hline Dwell Period (@50 m GIFOV) & $7.25 \mathrm{~ms}$ & \\
\hline Polarisation Control & Polarisation scrambler & With telescope mirror configuration compensation. \\
\hline Spectral Image Slit & At spectrometer focal plane & $\begin{array}{l}\text { Potentially dual slit (one for each detector) (UV can } \\
\text { be larger GIFOV and co-registration not as critical) }\end{array}$ \\
\hline
\end{tabular}
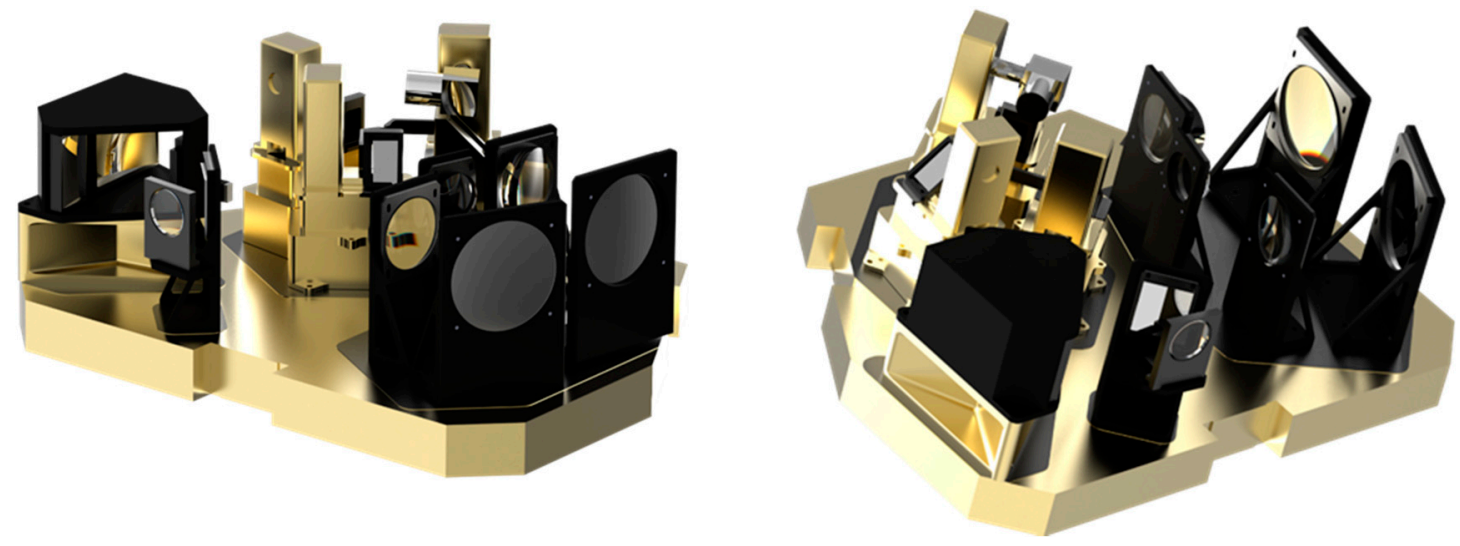

Figure 3. HIS on optical bench. Two views of an initial Surrey Satellite Technology Ltd (SSTL) concept design, which is subject to change as the mission undergoes detailed design during a formal Phase AB1.

\subsubsection{Cryogenic Solar Absolute Radiometer (CSAR)}

The heart of the calibration system is the primary SI standard, the cryogenic solar absolute radiometer (CSAR). CSAR is a flight version of the primary standard cryogenic, adopted by most NMIs worldwide and recommended as the means to achieve SI traceability [31]. The cryogenic radiometer is an extension of the electrical substitution radiometer (ESR) concept, typically cooled terrestrially to $<\sim 30 \mathrm{~K}$ reducing uncertainty levels to $<0.01 \%(k=2)$. Due to constraints with availability of space coolers with adequate performance CSAR will operate at a slightly higher temperature $<60 \mathrm{~K}$ (higher temperature increases the time constant and reduces sensitivity a little), when flown it will be the first cryogenic radiometer, and consequentially optical primary SI radiometric standard flown in space and is a key element of the calibration system of TRUTHS.

Most NMIs have adopted the cryogenic radiometer as the primary standard for radiometric measurements [32-34]. The cryogenic radiometer is an extension of the concepts of the electrical 
substitution radiometer (ESR), which were independently developed by the meteorologist Ångström (1893) [35] and the metrologist, Kurlbaum (1894) [36] and are in essence the basis of instruments currently flying in space measuring TSI, e.g., PMO6 on SOHO. Cooling the technology to cryogenic temperatures (originally $<30 \mathrm{~K}$ ) reduces uncertainty levels to $<0.01 \%(k=2)$ as first shown by NPL in the 1980s $[37,38]$. For TRUTHS, CSAR v2 will operate at $<60 \mathrm{~K}$ and provide traceability of Earth spectral radiance measurements to SI to an absolute accuracy of $<0.3 \%(k=2)$. In addition to serving as a primary standard for radiance and irradiance, CSAR will also provide science measurements in its own right, TSI with an accuracy of $0.02 \%(k=2)$, a significant improvement over most existing ambient temperature radiometers.

Because CSAR is both critical to the TRUTHS mission's ability to achieve its aims and the key novel aspect of TRUTHS, the instrument was first prototyped (CSAR_v1) in 2010 and has successfully undergone both laboratory and field tests (see below). A second version of the instrument (CSAR_v2), an evolved and simplified design, making direct use of an upgraded Airbus space cooler (currently flying on Sentinel 3) was built and tested together wth a breadboard of the full On-Board Calibration System (OBCS) in 2016. Note that because of the cryogenic components, all cryogenic radiometers are designed for vacuum operation and the CSAR_V1/2 can be considered TRL 5/6.

A cryogenic radiometer is an ESR that is cooled down to cryogenic temperatures. Figure 4 illustrates the operating principle of ESRs. A cavity that is connected to a heat sink via a thermally weak heat link is first heated up by incoming optical radiation (Figure 4, left hand side); subsequently, the radiation is blocked and the cavity is electrically heated to the same temperature (Figure 4, right hand side). This procedure allows the determination of radiant power through the measurement of electrical power applied to the heater. The thermometer serves only as a null detector and thus it is only short-term stability and resolution that are factors that need to be considered. Figure 5 illustrates how the radiometer and cooler integrate, and the link between the compressor and cold head is flexible, allowing their respective locations and orientations to be independently selected.

The main reason for cooling the radiometer is that the cavity can be larger than one at ambient temperature, whilst keeping the natural time constant similar and maintaining an adequate sensitivity. This is due to the much-reduced heat capacity of the cavity material (copper) at low temperatures. A physically larger cavity has much higher absorptivity, with less sensitivity to any degradation of the black surface coating of the cavity. Being able to make the cavity larger also means that for the TSI measurements, the larger FOV limiting aperture can be placed in front of the cavity instead of at the front of the instrument; with significantly reduced bias due to stray light caused by interaction of the light with the front aperture. Other reasons for operating the detector at low temperatures are reduced background radiation and elimination of bias in the electrical substitution by using superconducting leads to the electrical heater.

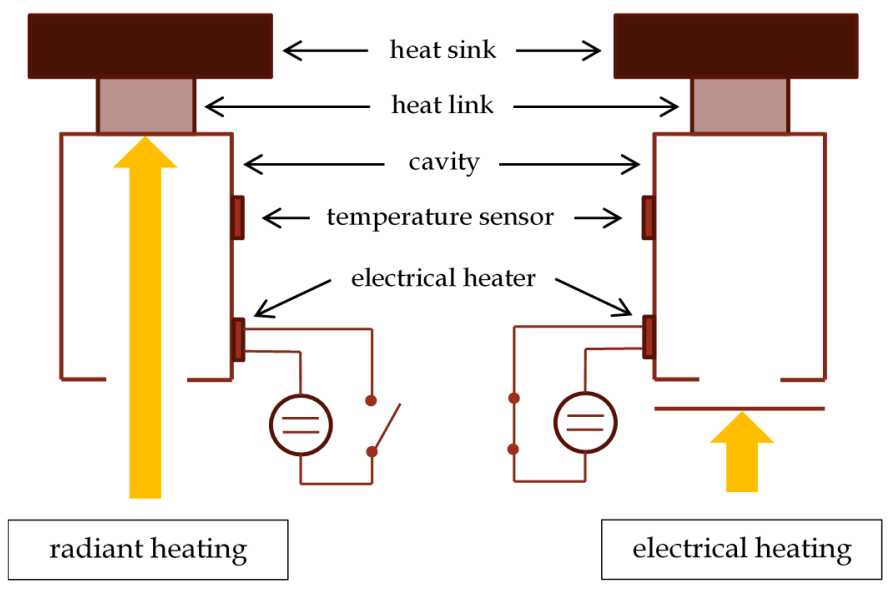

Figure 4. Operating principle of electrical substitution radiometers. 


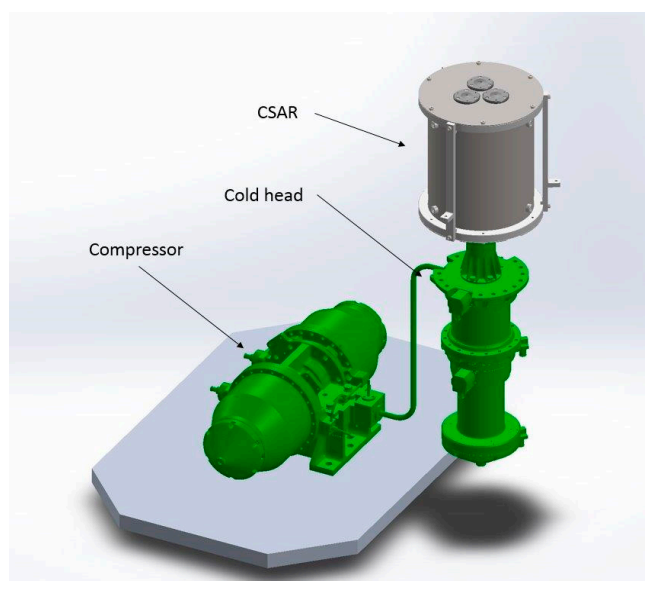

Figure 5. Schematic diagram showing overall layout for the TRUTHS cryogenic solar absolute radiometer (CSAR).

A CSAR-V1, built in 2010 and tested as a fully working 'engineering model' for TRUTHS, currently operates in Davos as a WMO world reference for terrestrial TSI measurements. A more optimised (low mass) flight representational CSAR-V2, fully coupled to a flight demonstrated space cooler, has more recently been built and tested with an Airbus high-power Stirling cooler (HPSC) space cooler see Figure 6.

(a)

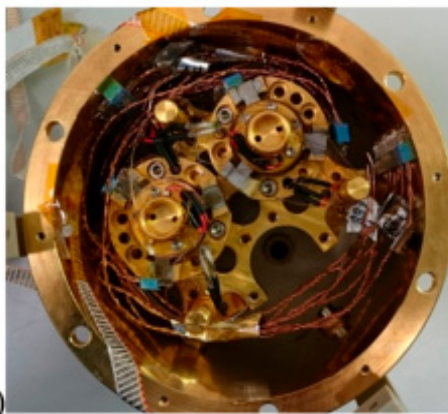

(b)

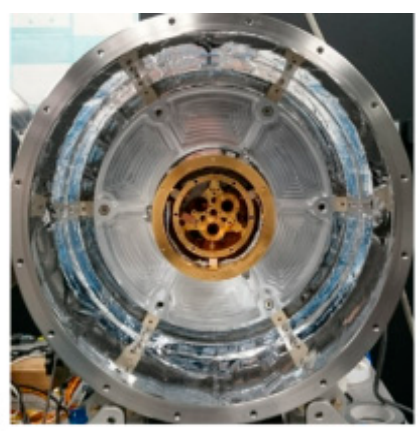

(c)

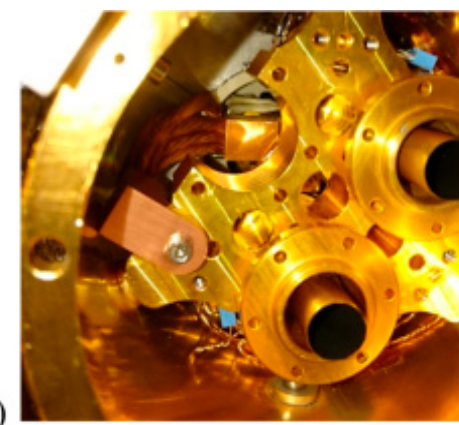

Figure 6. Photographs of the assembly of CSAR_V2 showing, from left to right, (a) two cavities mounted on the temperature reference block $(\mathbf{b})$ the radiometer head coupled to the high-power Stirling cooler (HPSC) and mounted inside the cryostat and terrestrial vacuum can (c) close up of cavities showing thermal link to the HPSC.

One of the principles underpinning CSAR's ability to establish SI-traceability in-flight is that the only optical interface that is subject to potential change is a blackened cavity. The use of a carbon nanotube black coating with resultant absorptance of $>0.99995$ makes it inherently insensitive to change. This is further enhanced through the inclusion of three cavities for redundancy and to limit any exposure to sunlight for at least one cavity to minimise any ageing effects. The measurement electronics of CSAR are based on modified versions of existing flight systems used for TSI measurements and e.g., the recent CLARA on NorSAT 1. Earlier versions of the electronics have shown to be stable to the uncertainties required. Independently, Airbus have performed a reliability study (five- and seven-year timeframes) on key electronic components and indicated that existing qualified components meet the requirement even for the extended lifetime.

\subsubsection{On-Board Calibration System (OBCS) Overview}

Central to the TRUTHS philosophy is the calibration system which puts SI traceability into orbit. The OBCS mimic the steps used by NMIs around the world to transfer calibration traceability 
from the SI defining power measurement (the cryogenic radiometer) to a full spectrally resolved radiance calibration of an instrument, spectrometer/radiometer, etc. The calibration system designed for TRUTHS has been simplified relative to those used at NMIs to minimise the number of components, and in particular, the number of moving parts compared to measurements in the laboratory and also to reflect the use, where possible, of heritage space components or heritage constituent technologies (Figure 7).
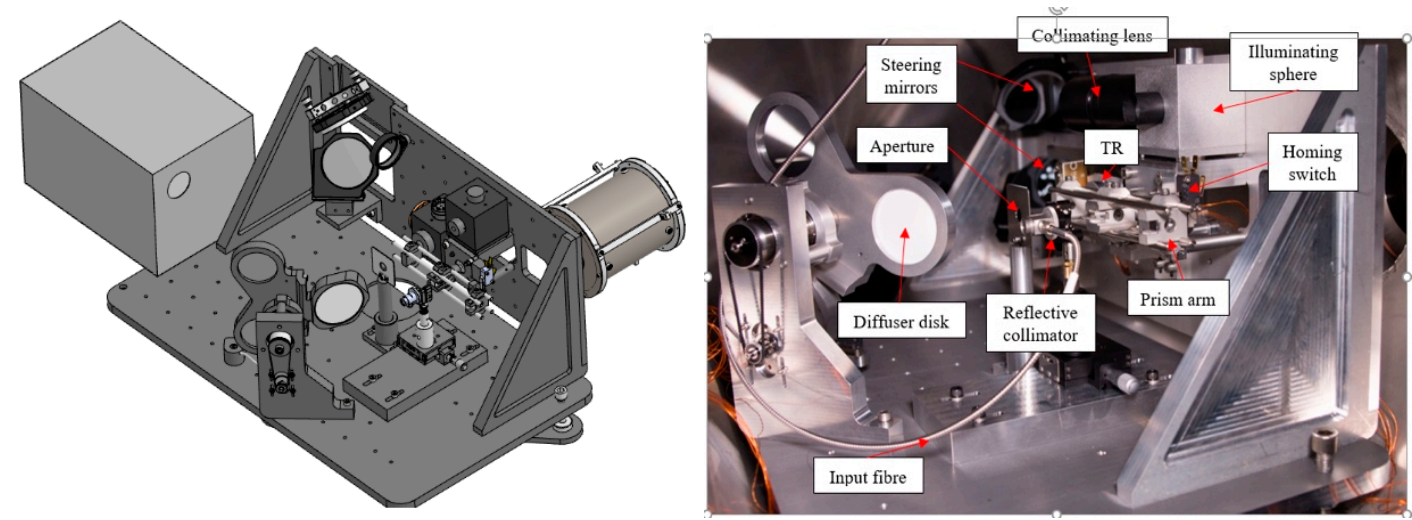

Figure 7. Computer-aided design drawing of laboratory breadboard of the TRUTHS OBCStogether with a photograph of its engineering realisation before testing in a vacuum tank.

The calibration of the HIS for Earth radiance and SSI measurements is performed, traceably to the CSAR, using a system that consists of a transfer radiometer (TR), a small number of ( 8-10) low power laser diodes (LDs), an integrating sphere (IS), and a diffuser wheel with two diffusers (D1, D2) for redundancy. Calibration generally takes place during the Earth shadow part of the orbit allowing the systems to be well-protected from solar and other radiation damage. However, during the commissioning phase at least, evaluations of the calibration process will be undertaken at various parts of the orbit including sun-lit and for nominal pointing configurations of the platform.

The full OBCS together with the CSAR v2, shown in Figure 7, has been fully demonstrated under vacuum and is ready for implementation and can largely be considered as TRL 5/6. A lifetime analysis of the full calibration system has been carried out by Airbus resulting in a value of 0.97 for a five-year mission.

\subsubsection{On-Board Calibration Procedure}

Figure 8 describes the calibration sequence and a video representation can be found here [39]. This is performed with just two mechanisms within the calibration system. A diffuser wheel, common on heritage sensors, and a rotating laser arm are used to distribute the laser power into three of the calibration sub-systems.

Here, we discuss, in outline, the calibration steps. Note that the diagrams here are schematic, and in the actual flight implementation, for example, additional turning mirrors may be used, which do not affect the overall calibration principle. Similarly, the exact design of sub-systems such as transfer radiometer and laser illumination systems may also differ in detail. It is also likely that, without adding any additional rotational mechanisms, further redundancy can be achieved for all of the critical sub-systems.

An example layout used as the breadboard is shown in Figure 7 and in a more cartoon style in Figure 9. The primary calibration is performed at a small number of wavelengths corresponding to the chosen LDs. These LDs can illuminate either of two (identical) cavities of the CSAR (providing redundancy), the TR, and the IS (which is used as part of the illumination of the HIS during calibration). The LDs illuminate each instrument/sub-system in turn using an illumination system based on a rotating pair of rooftop prisms serving as mirrors, with the central axis (mirror) being fed by laser 
radiation from a common optical rod conjoiner linking LDs via optical fibre (Figure 9). An alternative realisation may involve the LDs or at least coupled fibres attached directly to the perimeter of the rotation wheel, avoiding the prism mirrors. The optical fibre in either case is wound around the compressor of the cryocooler of CSAR where the micro-vibrations helps to reduce the effect of speckle. The monochromatic radiation from the LDs illuminates the full aperture of HIS in a Lambertian manner via a flat plate diffuser in a similar manner to existing on-board systems, the difference being the use of monochromatic LD radiation rather than the Sun. The radiance of the diffuser can be measured directly inflight by the on-board calibrated TR. The responsivity at all other intermediate wavelengths is obtained using a white light source e.g., incandescent lamp, which is radiometrically anchored at the LD wavelengths. The following steps scope the calibration procedure, which mimics that carried out terrestrially. It should also be noted that in addition to radiometric calibration the use of LDs allows wavelength accuracy of HIS to be checked and also of particular interest, stray-light. The latter, whilst not having enough LDs to provide a full calibration matrix as can be done on ground using tuneable lasers, does provide sufficient spectral sampling to allow the pre-flight correction matrix to be at least monitored in flight.

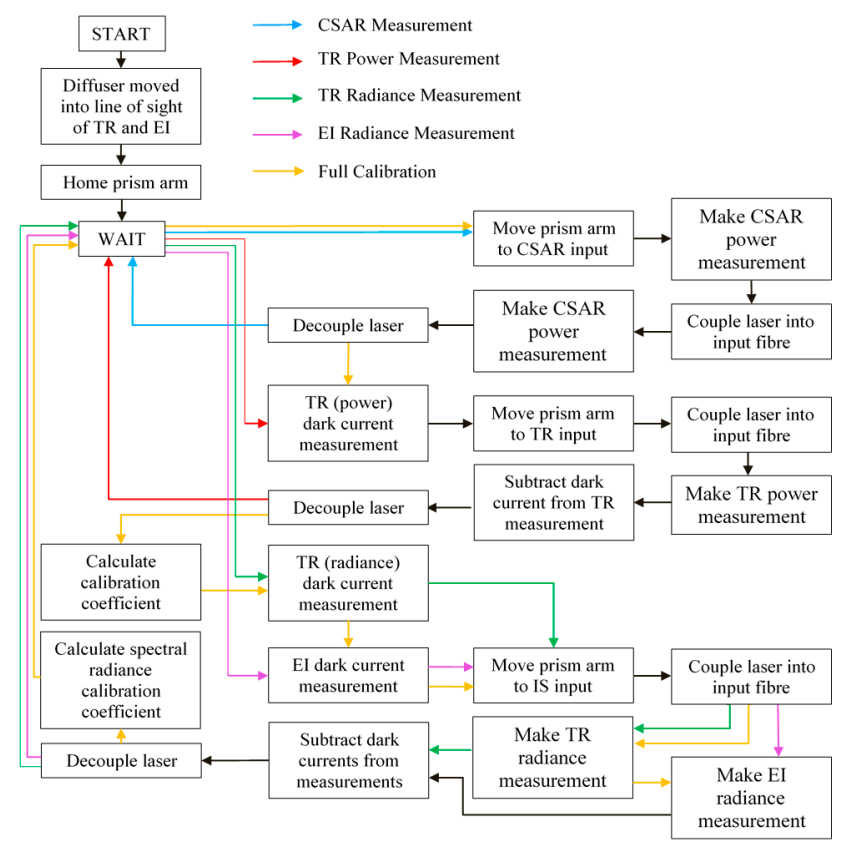

Figure 8. The TRUTHS calibration system calibration sequence.

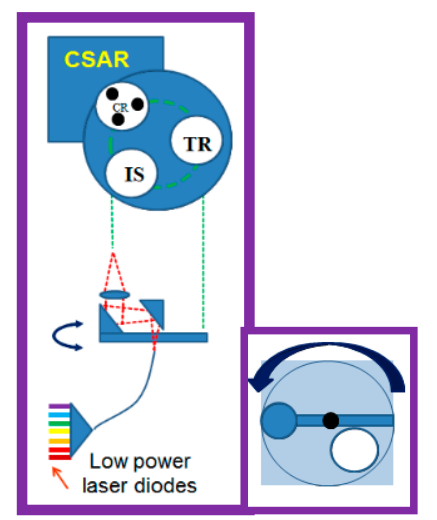

Figure 9. Laser diode (LD) illumination system (schematic). 
Step 1: Calibration of TR against CSAR using LDs.

The optical power of the temperature-and-current-stabilised LD is measured absolutely by the CSAR (Figure 10a) and the beam underfilling the CSAR irradiance defining aperture and then used to calibrate the response of the TR to this now measured power level by rotation of the prism assembly (Figure 10b). The TR in this illustration is a small integrating sphere with integral Si and InGaAs semiconductor photodiodes viewing its internal wall. The integrating sphere provides a convenient means to reduce any alignment or source non-uniformity issues (although alternative methods to achieve the same goal can be envisaged and may in practise be utilised). It has two field-of-view (FOV) defining apertures (radiance), but for this phase of the calibration, they are underfilled. This step is repeated for each of the LD wavelengths in turn. The calibration process requires the LD output to be of known wavelength (controlled with an etalon) and stable in intensity $(<0.1 \%)$ during the transfer from the CSAR to TR (about $1 \mathrm{~min}$, which is easily achieved). Individual wavelength calibrations can take place sequentially or on different orbits. However, it is probably likely to be optimum to perform the full calibration sequence step 1 and 2 for each wavelength individually.

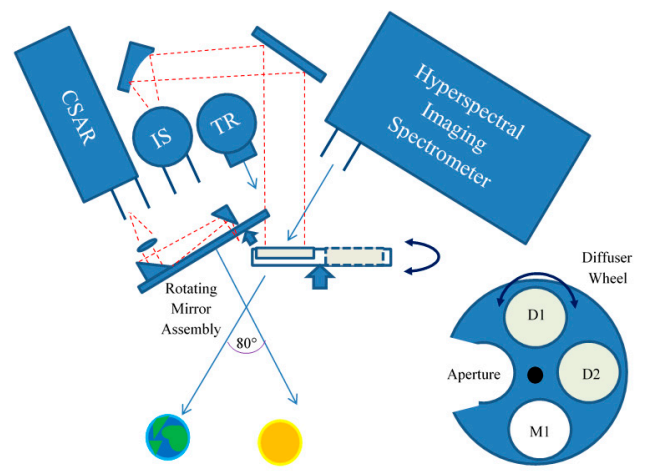

(a)

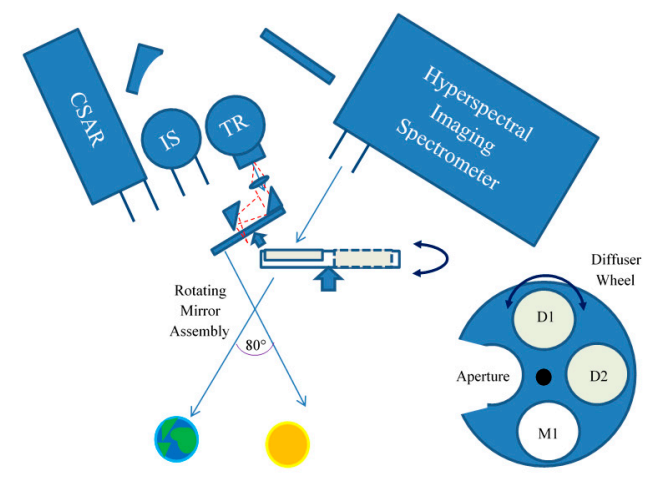

(b)

Figure 10. Schematic of step 1. The LDs illuminate (underfill) first (a) the CSAR cavity and then (b) the transfer radiometer (TR), by rotating the mirror assembly.

Step 2: HIS (Earth Radiance view) calibrated against the TR at each LD wavelength (radiance mode).

The radiance responsivity of the HIS is determined by comparison with the radiance responsivity of the TR using the LDs. In this step, the LD source is allowed to expand and then collimated to create a uniform illumination on a flat plate Lambertian diffuser, using an IS and mirrors (for alignment purposes). The IS not only helps to pre-condition the LD radiation, removing effects of non-uniformity, but also reduces significantly the effect of speckle, from both spatial and temporal coherence. The diffuser can move in and out of the FOV of the HIS by rotation of its mounting plate shown in Figure 11 with a second redundant diffuser and also mirror. When illuminated, normal to the diffuser as shown (Figure 11), the TR and HIS simultaneously view the diffuser at reciprocal angles.

In this step, the TR measures the absolute radiance of the LD-illuminated diffuser; its absolute radiance responsivity having been determined from its power responsivity (step 1) and knowledge of the areas of the two FOV-defining apertures, which are never directly exposed to the space environment. Note that, because the TR measures the radiance of the diffuser directly, the transmittance of the IS and reflectance of any turning mirrors (optics) do not need to be known. It is advantageous to limit the FOV differences between the TR and the HIS, but such differences and their small effect and can be characterised pre-flight and therefore an exact match is not required.

Thus, the HIS is calibrated for radiance responsivity at the LD wavelengths. Note also that by comparing the TR signals in Step 1 and Step 2 (assuming a stable laser source between these two measurements) the TR also directly calibrates the throughput of the IS, mirrors, and diffuser at these wavelengths. This provides an irradiance responsivity calibration of the system comprising the IS 
(underfilling an irradiance defining aperture on the entrance port), mirrors, diffuser, and HIS (this allows it to become the SSI measuring system, when the aperture is overfilled by solar radiation, see step 4).

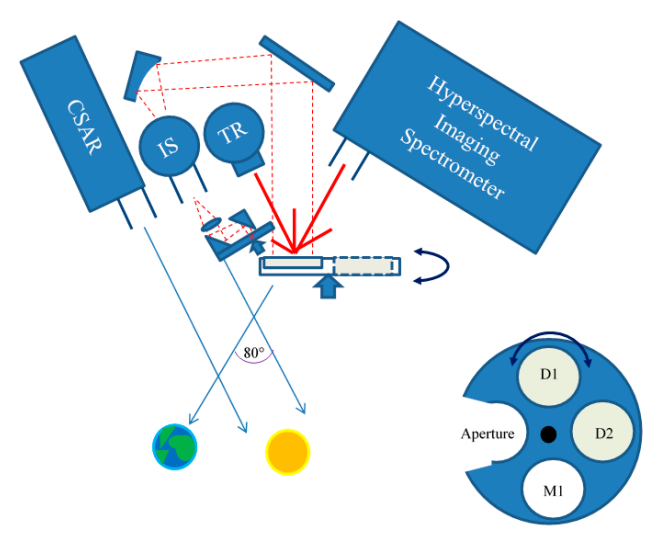

Figure 11. LD illuminates the diffuser, via the integrating sphere (IS), and this is viewed by the HIS and IS.

Step 3: HIS (Earth radiance view) calibrated at intermediate wavelengths with a lamp.

An incandescent lamp (with a smooth spectral output) illuminates the IS and consequently the diffuser and is viewed by the HIS in a similar manner to the LDs. At the end of step 2, the HIS has been calibrated at the wavelengths of the LDs for absolute radiance responsivity. It is now only necessary to interpolate between these laser wavelengths. This will be achieved using a white light source/lamp with a broadband, smooth spectral output. Although the lamp will change during operation, it will do so in a spectrally smooth manner. The absolute level and the basic spectral shape are determined at the LD wavelengths in step 2; this step (Figure 12) is primarily used for interpolation although at the extremes of the wavelength range of HIS, beyond the final anchoring wavelengths of the LDs the spectral shape is extrapolated. There are many examples of such lamps being used for space calibration, but here we only need functionality, short-term $(<10 \mathrm{~s})$ stability, and of course, spectrally smooth output.

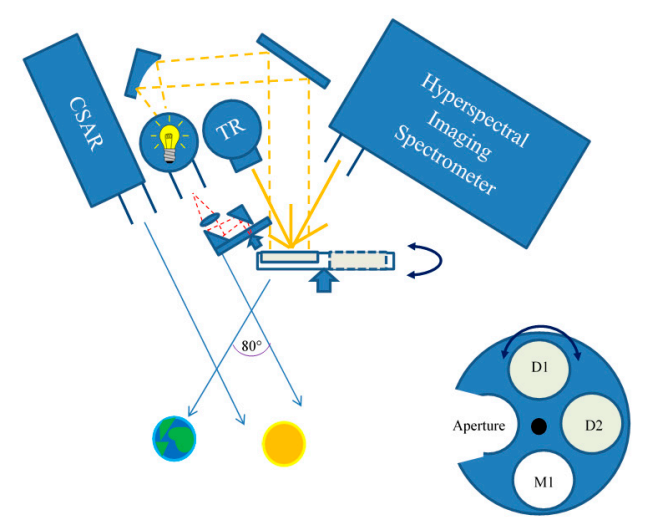

Figure 12. Lamp illuminates the diffuser via the integrating sphere and mirrors to provide calibration of the HIS at intermediate wavelengths.

The HIS can then be considered calibrated for making spectral radiance measurements of the Earth. Similarly, the system comprising the IS, mirrors, diffuser, and HIS can be considered calibrated for making spectral irradiance measurements of the sun/moon where the aperture on the IS is the defining aperture. 
Step 4: Measurements of the Earth and Sun

Measurements of the radiance of the Earth are made with the HIS directly. The diffuser disc rotates to allow light from the Earth to enter the HIS telescope (Figure 13). When the TRUTHS satellite is rotated to allow CSAR to face the Sun, then the CSAR can measure TSI and the IS (illuminating the mirrors, diffuser, and HIS) can measure SSI and similarly for the moon. For UV measurements of SSI, where the signal levels are very low, the mirror (M1) rather than diffuser (D1/D2) is used in the path between the IS and HIS.

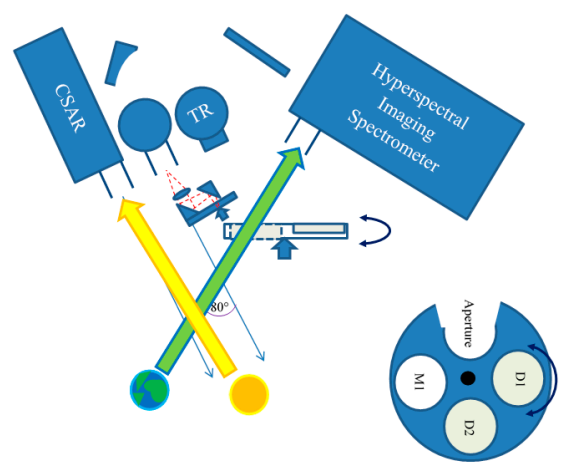

Figure 13. Measurements of the Earth and Sun.

These measurements are fully SI-traceable because of this calibration scheme for their radiometric responsivity. Full traceability to SI also needs traceability for the defining aperture areas (the two apertures on the TR, the aperture on the IS, and the aperture on CSAR). These apertures will be calibrated on the ground. In orbit, apertures on CSAR and IS will both be subject to direct exposure to space and the sun and so there is a need therefore to monitor the stability of those aperture areas. CSAR will have redundant apertures (different apertures on each cavity, which can have varying degrees of exposure). For the IS and in principle the TR, a dedicated LD beam (at one wavelength since geometry has no spectral dependency) is expanded with optics to a size that overfills the defining apertures of CSAR and the IS. By illuminating each CSAR aperture (redundant options) in turn, it can compare these apertures and calibrate the IS in irradiance (rather than power) mode. The same LD irradiance beam can also be used to check the smaller TR aperture as a relative comparison. It is also envisaged that there will be two IS' on TRUTHS each with their own aperture and again these will be used in a manner that allows reduced solar exposure time for one of the redundant pair.

Calibration procedure summary.

Figure 14 shows a schematic representation of the above calibration sequence for TRUTHS. Each complete iteration through the cycle (single wavelength) is estimated to take, at most, $5 \mathrm{~min}$ including time for any motions and stabilisation of the system.

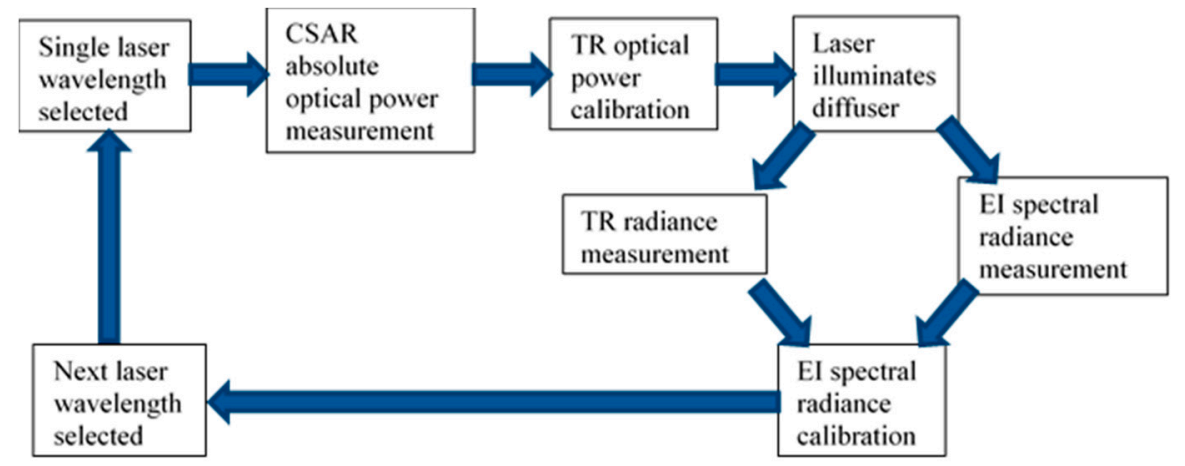

Figure 14. Schematic showing overall calibration sequence. 
All the sub-components of the OBCS, IS, TR, diffusers, detectors, and optical fibres can in themselves suffer degradation. The effects of this are removed by the calibration process, which effectively spectrally calibrates each component as part of the system. The Cal process relies on short term radiometric (power) stability of the LD radiation, which is largely related to temperature and drive current, both of which can be readily achieved for the $<1$-min timescales required. Wavelength stability or knowledge to $0.1 \mathrm{~nm}$ is required for the life of the mission. The calibration system, with the exception of the CSAR and the IS, are in an enclosed environment and never exposed to direct sunlight. Although in this baseline design only LDs, diffuser plate, and cavities of the CSAR are fully redundant, this is likely to change in the flight version and most of the sub-systems are also passive in nature, reducing failure risk; the exception being a rotation of the diffuser (similar to most Solar reflective EO imagers) and additionally the movement of laser radiation between the OBCS components.

\subsubsection{Payload Performance}

Table 9 shows the uncertainty budget for the calibration system, comparing the traditional NMI laboratory-based performance with the TRUTHS OBCS breadboard to the expected flight model performance. This table presents the principle sources of uncertainty in the calibration process of the HIS but does not account for any potential residual stray-light or non-linearity of the HIS. The latter will be evaluated by varying calibration radiance levels (laser diode power) and stray light will be evaluated as part of the calibration using the monochromatic wavelengths on-board to monitor the more detailed pre-flight characterisation using a full spectral calibration with a tuneable laser facility. The OBCS is designed to be non-polarised-the LDs themselves, the optical fibres, and scatter from the walls of the IS and diffusers ensuring that this is the case and so no component has been included for this in the uncertainty budget.

Figure 15 shows the progression of uncertainty propagation, with typical uncertainty budgets from the SI-traceable CSAR instrument, through to the HIS on-board calibration, sensor-to-sensor SNO cross calibration (see Table 6), and final example L2 product upgrade for the TRUTHS mission.

Table 9. Calibration system and HIS radiometric uncertainty $(\mathrm{k}=2)$.

\begin{tabular}{|c|c|c|c|c|}
\hline $\begin{array}{l}\text { Uncertainty } \\
\text { Source }\end{array}$ & $\begin{array}{l}\text { Lab-Based } \\
\text { Uncertainty }\end{array}$ & $\begin{array}{l}\text { Breadboard-Based } \\
\text { Uncertainty }\end{array}$ & $\begin{array}{l}\text { Space-Based } \\
\text { Uncertainty }\end{array}$ & Notes \\
\hline $\begin{array}{l}\text { Laser (diodes) } \\
\text { Radiometric } \\
\text { Stability (60 s) }\end{array}$ & $0.01 \%$ & $0.07-0.3 \%$ & $<0.07 \%$ & $\begin{array}{l}\text { Space qualified, stabilized DFB lasers. } \\
\text { Note for the breadboard testing not all } \\
\text { lasers were of DFB quality resulting in } \\
\text { poor stability }\end{array}$ \\
\hline CSAR/Primary Std & $0.02 \%$ & $0.06 \%$ & $<0.06 \%$ & $\begin{array}{l}\text { Worst-case scenario for laser diode } \\
\text { power levels }\end{array}$ \\
\hline Prism Arm & $<0.01 \%$ & $<0.01 \%$ & $<0.01 \%$ & Negligible \\
\hline $\begin{array}{c}\text { Transfer } \\
\text { Radiometer }\end{array}$ & $0.02 \%$ & $0.03 \%$ & $<0.03 \%$ & Voltage measurement uncertainty \\
\hline External Aperture & $0.02 \%$ & $0.02 \%$ & $0.02 \%$ & Calibrated pre-flight \\
\hline Traceability & $0.01 \%$ & $0.08 \%$ & $<0.08 \%$ & $\begin{array}{c}\text { Worst-case scenario and largely due } \\
\text { to potential residucal } \\
\text { non-uniformity effects }\end{array}$ \\
\hline $\begin{array}{l}\text { HIS Thermal } \\
\text { Control (1K) }\end{array}$ & & & $0.01-0.20 \%$ & $\begin{array}{c}<0.05 \% \text { at all wavelengths, except at } \\
\text { extremes }(<350 \mathrm{~nm} \&>2200 \mathrm{~nm})\end{array}$ \\
\hline HIS SNR & & & $0.03 \%$ & $\begin{array}{l}\text { Required laser source integration } \\
\text { times from } 7-60 \mathrm{sec} \text {, and some spatial } \\
\text { binning for solar spectral irradiance }\end{array}$ \\
\hline TOTAL & $0.04 \%$ & $0.16-0.32 \%$ & $0.16-0.26 \%$ & Uncertainties added in quadrature \\
\hline
\end{tabular}




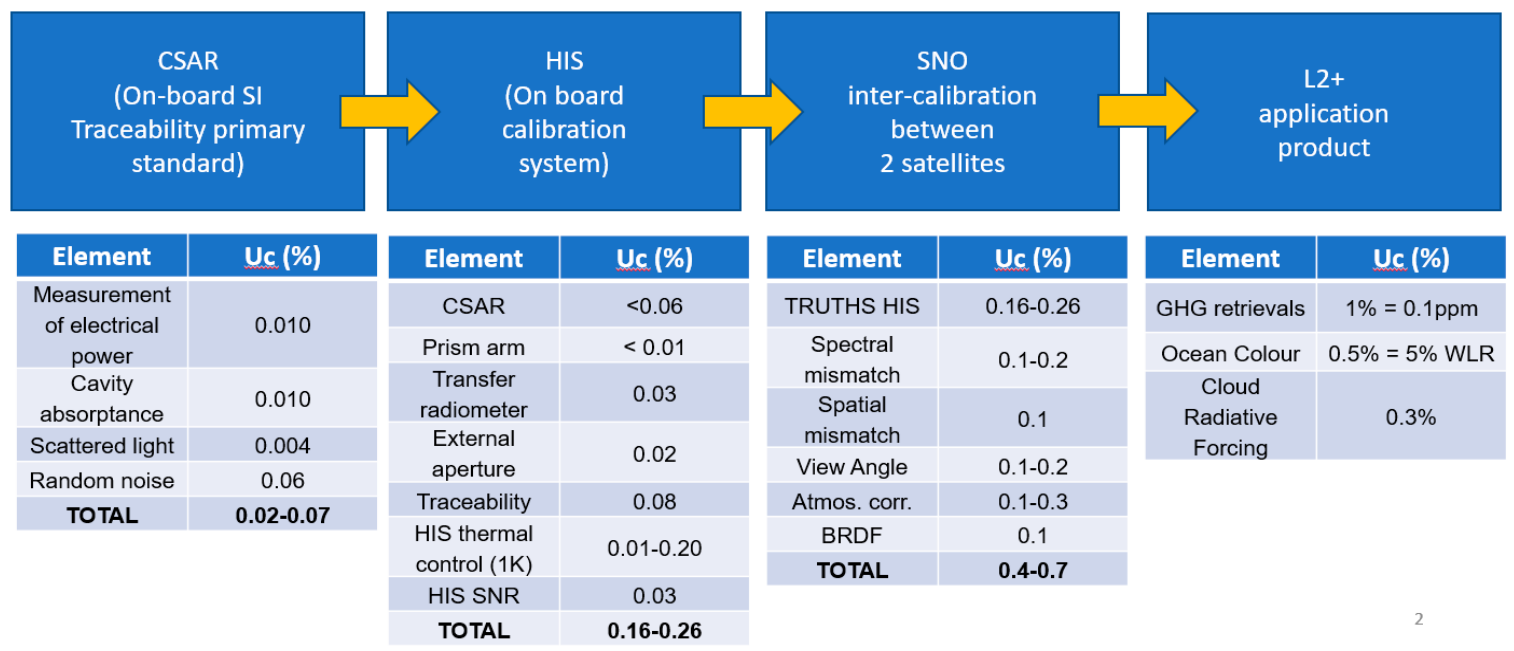

Figure 15. Uncertainty $(\mathrm{k}=2)$ propagation to inter-calibration and L2+ products.

\subsection{Satellite Bus and Launch}

A TRUTHS satellite platform has been defined and specified independently by both SSTL and Airbus to demonstrate the achievability using largely existing and heritage designs see Figure 16. The baseline system is an Airbus Astrobus-S although the M platform is also viable and the main alternative option is the SSTL spacecraft based on that used for Formosat 7, but there are alternatives.

A relatively detailed analysis, particularly related to avionics, for a low-cost platform has been carried out by SSTL and a similar one for the Astrobus-S but this will now be undertaken with greater rigour as part of on-going Phase AB1 studies and so not reported here.

(a)

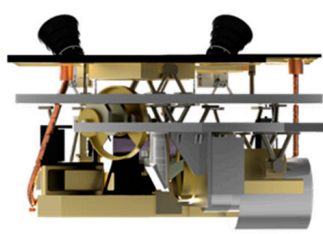

(b)

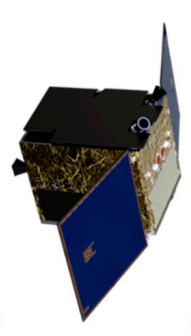

(c)
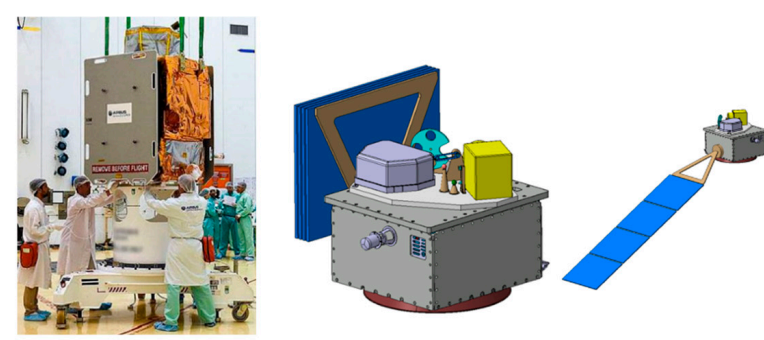

Figure 16. Images of possible platforms and payload integration: (a) payload integrated on SSTL Formosat 7 platform, (b) Formosat 7 candidate platform, (c) Peru-Sat1 on Airbus Astrobus-S (baseline platform for TRUTHS), (d) TRUTHS payload configured on Astrobus-S.

\subsection{Operational Modes}

Table 10 provides an outline of the observational plan for TRUTHS. The full calibration mode indicated to nominally take place bi-monthly will vary from beginning to end of life as a degradation profile is established. This full calibration mode includes all wavelengths traceable back to the CSAR and will be carried out in a continuous cyclic manner. However, calibrations of the imager can take place daily using coefficients of the calibration system established on an ongoing basis. Not all spectral channels need to be calibrated each cycle unless significant degradation is found, and similarly, at least one wavelength can be done per orbit on the earth shadow side of the orbit. On occasions, particularly during commissioning phase, some HIS calibrations at some wavelengths are likely to be carried out to assess any intra-orbital and or pointing effects. 
Table 10. TRUTHS operational modes.

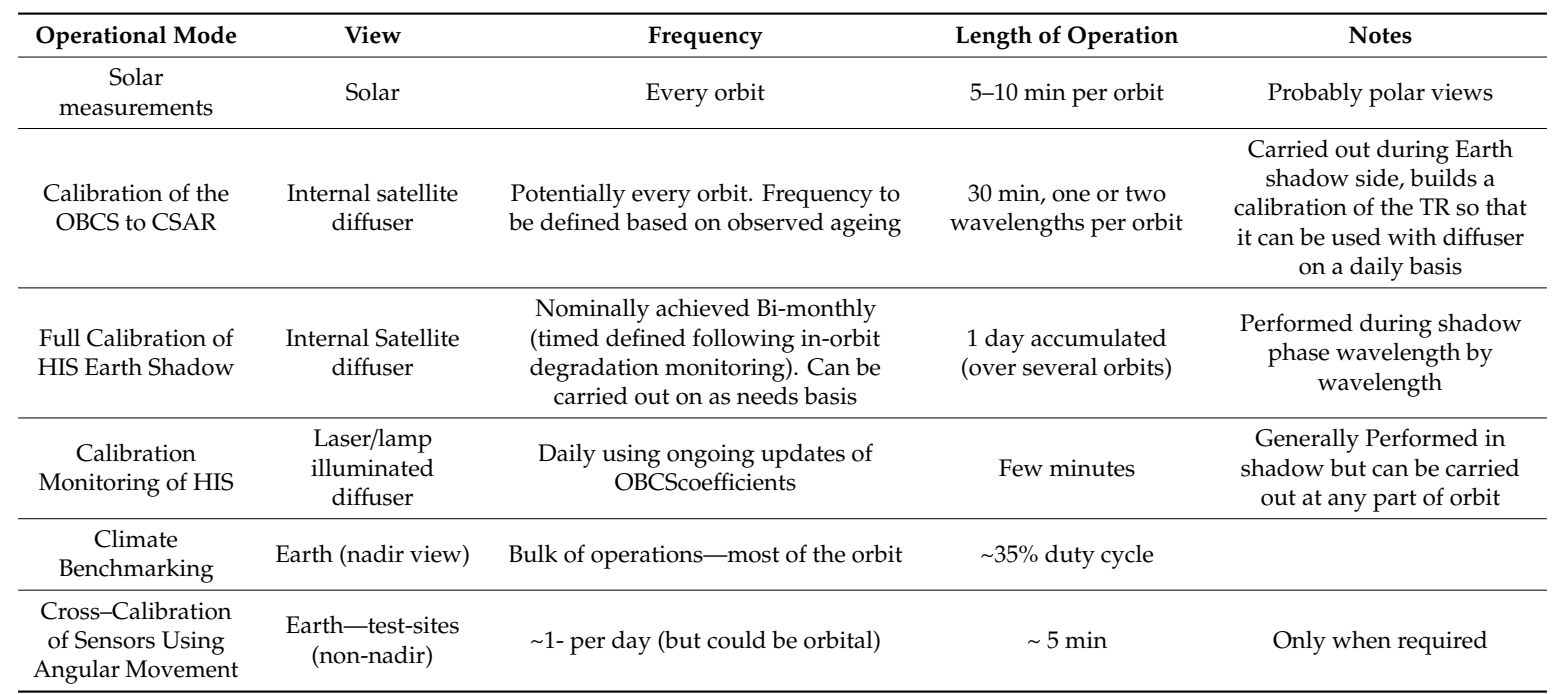

\section{Conclusions}

Climate change is the most critical issue facing mankind today. The enormous cost implications of policy decisions based on forecasted impacts resulting from the predictions of a warming Earth demand that the science community finds and delivers the necessary information with the highest possible confidence in the shortest possible time. The challenge to the metrology community is equally severe.

The IPCC [1] concludes that the mix of natural variability and anthropogenic effects on decadal time scales is far from fully understood or measured, requiring significant improvements in accuracy. Unequivocal attribution and quantification of subtle fingerprint indicators from this noisy background are fundamental to our ability to predict climate reliably and use appropriate mitigation/adaptation strategies. The uncertainty in climate prediction lies in the complexity of the models, our inadequate understanding of the Earth system and its feedback mechanisms, and the relatively poor quality of available data against which to test predictions on the necessary decadal time scales.

Establishing rigorous SI-traceability to the key measurands underpinning the ECVs, with sufficient accuracy, is a central pillar to achieving this goal. The satellite community has over the years developed a number of strategies to support this objective, but as yet all fall dramatically short of the required accuracy, forcing the adoption of high-risk philosophies seeking to monitor 'change' through normalization of overlapping datasets. While pre-flight calibrations can be made traceable, the harsh environment of space following the shock of launch means that few, if any, of the radiometric ground calibrations can be relied upon in space. Efforts to recover some of the information can be carried out using a variety of post-launch methods, but none have sufficient accuracy to meet the needs of climate [21].

This paper has described an example of the only real solution available to the EO community, i.e., to establish high-accuracy SI traceability in flight, on-board the spacecraft. One mission, TRUTHS, which is designed to achieve this for the solar-reflective domain, has been described in some detail. Its sister CLARREO and its pathfinder demonstrator complements this in the solar-reflective domain and extends the capability into the IR spectral region, and a similar Chinese mission is also under-development.

TRUTHS would become 'a standards laboratory in space' providing directly traceable measurements of unprecedented accuracy (factor of 10 improvement) in SI units, in orbit, through the deployment of a primary radiometric standard and associated calibration methodology. This unprecedented accuracy will establish benchmark measurements for the detection of decadal change in the most sensitive (but least understood) climate radiative forcings, responses, and feedbacks in the solar reflective domain. 
With spectrometer resolution sensors of unprecedented accuracy, TRUTHS can observe climate-relevant processes related to the atmosphere, the oceans, and the land surface. TRUTHS' observations will test and advance the development of climate models, allowing more accurate climate change hindcasting and forecasting. In addition, TRUTHS' measurements of ground-based reference sites (and the Moon) will allow retrospective improvements to the calibration of existing satellites and their data.

TRUTHS/CLARREO will provide an in-orbit standard for reference intercalibration for other EO satellite instruments including constellations of microsats (with benefits for both science applications and operational services). The TRUTHS and CLARREO missions are planned to fly in an orbit that allows frequent 'cross-overs' with other in-flight sensors. Together with their own pointing capabilities, and ability to match spectral and spatial resolution, the high calibration accuracy and SI-traceability will transfer to other sensors through simultaneous observations of a target.

SI-traceable observations at climate change accuracy from different instruments can then be objectively linked to a common baseline time-series even if no sensor-to-sensor overlaps are available. Had an in-orbit calibration observatory similar to TRUTHS/CLARREO already flown, this benefit would exist now. Delaying the installation of such a system reduces the value of the climate records and creates the constant risk of data records being lost completely should an overlap be lost. The full value of TRUTHS/CLARREO will be gained in the future, when a series of small platform in-orbit calibration instruments anchor EO time series, providing the highest possible confidence in any observed trends and at relatively low cost.

The realization of SI-traceable measurements in orbit is achievable now and time critical. This 'grand challenge' project between the metrology and Earth/climate science community must become a priority if society is to reap the commercial benefits of EO, but more fundamentally, without it, policymakers will be acting blindly in their efforts to ensure long-term sustainability and growth within an ever-changing climate.

Author Contributions: The original concept, methodology and design stems from N.F., with additional contributions from PG in developing the methodology, design and its practical realisation as an engineering demonstrator. The writing and editing of this paper was led by NF with contributions from P.G. All authors have read and agreed to the published version of the manuscript.

Funding: This research was funded by National Measurement system of BEIS, UK and also UK Space Agency through its Center for Earth Observation Instrumentation (CEOI) together with the project MetEOC-3 (16ENV03) of the European Metrology Programme for Innovation and Research (EMPIR). The EMPIR is jointly funded by the EMPIR participating countries within EURAMET and the European Union's Horizon 2020 program.

Acknowledgments: The authors would like to acknowledge contributions in developing the science case and requirements from the TRUTHS science team and in particular Bruce Wielicki and the CLARREO team and Professor Werner Schmutz former director of PMOD in the context of solar irradiance. In addition, Rainer Winkler, Lydia Zajiczek and Theo Hobson (at the time of NPL) for their efforts in building and testing the CSAR and OBCS.

Conflicts of Interest: Conflicts of interest: The authors declare no conflicts of interest

\section{References}

1. IPCC. Climate Change 2013: The Physical Science Basis. Contribution of Working Group I to the Fifth Assessment Report of the Intergovernmental Panel on Climate Change; IPCC: Cambridge, UK, 2013.

2. NRC. Earth Science and Applications from Space: National Imperatives for the Next Decade and Beyond; National Academy Press: Washington, DC, USA, 2007.

3. Wielicki, B.A.; Young, D.F.; Mlynczak, M.G.; Thome, K.J.; Leroy, S.; Corliss, J.; Anderson, J.G.; Ao, C.O.; Bantges, R.; Best, F.; et al. Achieving Climate Change Absolute Accuracy in Orbit. BAMS 2013. [CrossRef]

4. Essential Climate Variables. Available online: https://gcos.wmo.int/en/essential-climate-variables (accessed on 26 June 2020).

5. Huang, Y.; Leroy, S.; Gero, J.; Dykema, J.; Anderson, J. Separation of longwave climate feedbacks from spectral observations. J. Geophys. Res. 2010, 115. [CrossRef] 
6. Muscheler, R.; Joos, F.; Beer, J.; Müller, S.A.; Vonmoos, M.; Snowball, I. Solar activity during the last 1000 yr inferred from radionuclide records. Quat. Sci. Rev. 2007, 26, 82-97. [CrossRef]

7. Vonmoos, M.; Beer, J.; Muscheler, R. Large variations in Holocene solar activity: Constraints from Be-10 in the Greenland Ice Core Project ice core. J. Geophys. Res. Space Phys. 2006, 111, A10. [CrossRef]

8. Schatten, K.H.; Orosz, J.A. Solar-Constant Secular Changes. Sol. Phys. 1990, 125, 179-184. [CrossRef]

9. TSI composite. Available online: http://www.pmodwrc.ch/pmod.php?topic=tsi/composite/SolarConstant (accessed on 26 June 2020).

10. Ineson, S.; Maycock, A.C.; Gray, L.J.; Scaife, A.A.; Dunstone, N.J.; Harder, J.W.; Knight, J.R.; Lockwood, M.; Manners, J.C.; Wood, R.A. Regional climate impacts of a possible future grand solar minimum. Nat. Commun. 2015, 6, 7535. [CrossRef]

11. Menang, K.P.; Coleman, M.D.; Gardiner, T.D.; Ptashnik, I.V.; Shine, K.P. A high-resolution near-infrared extraterrestrial solar spectrum derived from ground-based Fourier transform spectrometer measurements. J. Geophys. Res. Atmos. 2013, 118, 5319-5331. [CrossRef]

12. CLARREO Science Team Report. Available online: https://clarreo.larc.nasa.gov/pdf/CLARREO_Science_ Team_Report_final.pdf (accessed on 26 June 2020).

13. Haigh, J.D. The effects of solar variability on the Earth's climate. Philos. Trans. R. Soc. Lond. A Math. Phys. Eng. Sci. 2003, 361, 95-111. [CrossRef]

14. Feldman, D.R.; Algieri, C.A.; Collins, W.; Roberts, Y.; Pilewskie, P. Simulation studies for the detection of changes in broadband albedo and shortwave nadir reflectance spectra under a climate change scenario. J. Geophys. Res. 2011. [CrossRef]

15. Feldman, D.R.; Collins, W.D.; Paige, J.L. Pan-spectral observing system simulation experiments of shortwave reflectance and long-wave radiance for climate model evaluation. Geosci. Model. Dev. 2015, 8, 1943-1954. [CrossRef]

16. Wielicki, B.A.; Parker, L. On the Determination of Cloud Cover from Satellite Sensors: The Effect of Sensor Spatial Resolution. J. Geophys. Res. 1992, 97, 12-799. [CrossRef]

17. European Commission. A European Research and Innovation Roadmap for Climate Services; European Commission: Brussels, Belgium, 2015.

18. Cooke, R.; Wielicki, B.; Young, D.B.; Mlynczak, M.G. Value of information for climate observing systems. Environ. Syst. Decis. 2014, 34, 98-109. [CrossRef]

19. Radiometric Calibration Network Portal. Available online: http://www.radcalnet.org (accessed on 26 June 2020).

20. Bouvet, M.; Thome, K.; Berthelot, B.; Bialek, A.; Czapla-Myers, J.; Fox, N.P.; Goryl, P.; Henry, P.; Ma, L.; Marcq, S.; et al. RadCalNet: A Radiometric Calibration Network for Earth Observing Imagers Operating in the Visible to Shortwave Infrared Spectral Range. Remote Sens. 2019, 11, 2401. [CrossRef]

21. Chander, G.; Hewison, T.; Fox, N.; Wu, X. Overview of Intercalibration of Satellite Instruments. IEEE Trans. Geosci. Remote Sens. 2013, 51, 1056-1080. [CrossRef]

22. Boussole: Buoy for the Acquisition of Long-Term Optical Time Series. Available online: http://www.obs-vlfr. fr/Boussole/html/cruises/monthly.php (accessed on 26 June 2020).

23. Stone, T.C.; Kieffer, H.H. Assessment of Uncertainty in ROLO Lunar Irradiance for On-orbit Calibration. Proc. SPIE 2004, 5542, 300-310.

24. Chander, G.; Helder, D.L.; Aaron, D.; Mishra, N.; Shrestha, A.K. Assessment of Spectral, Misregistration, and Spatial Uncertainties Inherent in the Cross-Calibration Study. IEEE Trans. Geosci. Remote Sens. 2013, 51, 1282-1296. [CrossRef]

25. Gorroño, J.; Banks, A.C.; Fox, N.P.; Underwood, C. Radiometric inter-sensor cross-calibration uncertainty using a traceable high accuracy reference hyperspectral imager. ISPRS J. Photogramm. Remote Sens. 2017, 130, 393-417. [CrossRef]

26. Global Climate Observing System: Systematic Observation Requirements for Satellite-Based Data Products for Climate: 2011 Update; GCOS-154; WMO: Geneva, Switzerland, 2011; p. 128.

27. Zibordi, G.; Melin, F.; Voss, K.; Johnson, B.C.; Franz, B.; Kwiatkowska, E.; Huot, J.P.; Wang, M.; Antoine, D. System vicarious calibration for ocean color climate change applications: Requirements for in situ data. Remote Sens. Environ. 2015, 159, 361-369. [CrossRef]

28. Lunar Calibration: ROLO Robotic lunar Observatory. Available online: https://astrogeology.usgs.gov/moon$\mathrm{cal} /$ (accessed on 26 July 2020). 
29. Fox, N.; Aiken, J.; Barnett, J.J.; Briottet, X.; Carvell, R.; Frohlich, C.; Groom, S.B.; Hagolle, O.; Haigh, J.D.; Kieffer, H.H.; et al. Traceable Radiometry Underpinning Terrestrial- and Helio- studies (TRUTHS). Adv. Space Res. 2003, 32, 2253-2261. [CrossRef]

30. Fox, N.; Kaiser-Weiss, A.; Schmutz, W.; Thome, K.; Young, D.; Wielicki, B.; Winkler, R.; Woolliams, E. Accurate radiometry from space: An essential tool for climate studies. Phil. Trans. R. Soc. A 2011, 369, 4028-4063. [CrossRef]

31. Mise en Pratique for the Definition of the Candela and Associated Derived Units for Photometric and Radiometric Quantities in the SI. Available online: https://www.bipm.org/utils/en/pdf/si-mep/SI-App2candela.pdf (accessed on 26 June 2020).

32. Fox, N.P.; Haycocks, P.R.; Martin, J.E.; Ul-Haq, I. A mechanically cooled portable cryogenic radiometer. Metrologia 1995, 32, 581-584. [CrossRef]

33. Gentile, T.R.; Houston, J.M.; Hardis, J.E.; Cromer, C.L.; Parr, A.C. National Institute of Standards and Technology high-accuracy cryogenic radiometer. Appl. Opt. 1996, 35, 1056-1068. [CrossRef] [PubMed]

34. Goebel, R.; Stock, M.; Köhler, R. Report on the International Comparison of Cryogenic Radiometers; BIPM Report. BIPM. Available online: https://www.bipm.org/utils/common/pdf/rapportBIPM/RapportBIPM-2000-09.pdf (accessed on 26 July 2020).

35. Ångström, K. Eine electrische Kompensationsmethode zur quantitativen Bestimmung strahlender Wärme. Nova Acta Soc. Sci. Upsal. Ser. 1893, 3, 16.

36. Kurlbaum, F. Notiz über eine Methode zur quantitativen Bestimmung strahlender Wärme. Ann. Phys. (Leipzig) 1894, 287, 591. [CrossRef]

37. Quinn, T.J.; Martin, J.E. A radiometric determination of the Stefan-Boltzmann constant and thermodynamic temperatures between $-40{ }^{\circ} \mathrm{C}$ and $+100{ }^{\circ} \mathrm{C}$. Philos. Trans. R. Soc. 1985, 316, 85-189.

38. Martin, J.E.; Fox, N.P.; Key, P.J. A cryogenic radiometer for absolute radiometric measurements. Metrologia 1985, 21, 147-155. [CrossRef]

39. TRUTHS, Traceable Radiometry Underpinning Terrestrial- and Helio- Studies. Available online: https: //youtu.be/__BznE1Ckgc (accessed on 26 June 2020).

(C) 2020 by the authors. Licensee MDPI, Basel, Switzerland. This article is an open access article distributed under the terms and conditions of the Creative Commons Attribution (CC BY) license (http://creativecommons.org/licenses/by/4.0/). 